\title{
Research Article \\ Characterizations of Tight Frame Wavelets with Special Dilation Matrices
}

\author{
Huang Yongdong ${ }^{1}$ and Zhu Fengjuan ${ }^{2}$ \\ ${ }^{1}$ Institute of Information and System Science, Beifang University of Nationalities, Yinchuan 750021, China \\ ${ }^{2}$ Department of Foundation, Beifang University of Nationalities, Yinchuan 750021, China
}

Correspondence should be addressed to Huang Yongdong, nxhyd74@126.com

Received 22 May 2010; Revised 29 October 2010; Accepted 25 November 2010

Academic Editor: Angelo Luongo

Copyright (c) 2010 H. Yongdong and Z. Fengjuan. This is an open access article distributed under the Creative Commons Attribution License, which permits unrestricted use, distribution, and reproduction in any medium, provided the original work is properly cited.

We study all generalized low-pass filters and tight frame wavelets with special dilation matrix $M$ (M-TFW), where $M$ satisfies $M^{d}=2 I_{d}$ and generates the checkerboard lattice. Firstly, we study the pseudoscaling function, generalized low-pass filters and multiresolution analysis tight frame wavelets with dilation matrix $M$ (MRA M-TFW), and also give some important characterizations about them. Then, we characterize all M-TFW by showing precisely their corresponding dimension functions which are nonnegative integer valued. Finally, we also show that an M-TFW arises from our MRA construction if and only if the dimension of a particular linear space is either zero or one.

\section{Introduction}

Wavelet analysis with its fast algorithms is used in many fields of applied mathematics, such as image or signal analysis and numerical treatment of operator equations (see [1-4]). Moreover, wavelet bases, recently also wavelet frames, are applied to the characterization of the function space [5]. The classical MRA wavelets are probably the most important class of orthonormal wavelets. Many of the better known examples as well as those often used in applications belong to this class. However, Journe's wavelet is not an MRA wavelet. Thus, it was a natural question to find necessary and sufficient conditions for an orthonormal wavelet to be an MRA wavelet. An interesting approach to this involves the dimension function. On the other hand, there are useful filters, such as $m(\xi)=(1 / 2)\left(1+e^{3 i \xi}\right)$, that do not produce orthonormal basis; nevertheless, they produce systems that have the reconstruction property, as well as many other useful features.

It is natural, therefore, to develop a theory involving more general filters that do, indeed, produce systems having these properties. A natural setting for such a theory is provided by frames [1]. Several authors have considered this problem and have shown how more general filters produce such frames. A successful development of these ideas is 
provided by the papers [6, 7]. These results, however, do involve certain restrictions and technical assumptions such as semiorthogonality. In particular, they exclude the use of the filter we described above. A related approach can be found in [8]. Note that the design of tight wavelet frames is still a challenging problem and a number of references have dealt with this subject (see [3-25]).

In $[20,21]$, authors successfully developed a theory of univariate tight frame wavelets and MRA tight frame wavelets. In [24], authors revealed the deep and rich structure of the set of dyadic tight frame wavelets. In [9, 10, 25], authors extended the one-dimensional case to the multidimensional case with the expending dilation matrix of determinant 2, but it is a pity that authors only studied the pseudoscaling function, generalized lowpass filters, and the multiplier classes associated with M-TFWs, but they did neither study the relationship between MRA M-TFW and M-TFW, nor study the relationship between MRA M-TFW and dimension function. In [18], authors constructed multivariate compactly supported tight wavelet frames with dilation matrix $2 I$, unfortunately, they obtained that at least $2^{d}-1$ tight frame generators can generate a tight wavelet frame. Therefore, with the dimension of space increasing, the computational complexity increases. In [8], the author gave the characterizations of the abstract tight frames with arbitrary dilation matrix for $L^{2}\left(\mathbb{R}^{d}\right)$; due to arbitrariness of dilation matrix, the author did not give the expression of tight frame generators (in the time domain or the frequency domain). In fact, even in onedimensional case, it is very difficult to give the explicit expression of tight frame generators with dilation factor $a(a>2)$ by means of scaling function (see [16]). With the dimension $d$ increasing, the computational complexity increases, with the absolute value of determinant of the dilation matrix $M$ increasing, the computational complexity increases, that is, at least $|\operatorname{det} M|-1$ generators can generate tight wavelet frames for $L^{2}\left(\mathbb{R}^{d}\right)$. Therefore, we have to recur some special dilation matrices to solve the problem in $L^{2}\left(\mathbb{R}^{d}\right)$. In the paper, we study all the generalized low-pass filters and tight frame wavelets with special dilation matrix $M$ (M-TFW), where $M$ satisfies $M^{d}=2 I_{d}$ and generates the checkerboard lattice [14], that is,

$$
M \mathbb{Z}^{d}=\left\{k \in \mathbb{Z}^{d}: \sum_{i=1}^{d} k_{i} \in 2 \mathbb{Z}\right\} .
$$

In this case, we only need one function $\psi$ such that the system $\left\{\psi_{j, k}, j \in \mathbb{Z}, k \in \mathbb{Z}^{d}\right\}$ is a tight frame (with $A=B=1$ ) for $L^{2}\left(\mathbb{R}^{d}\right.$ ) (in other word, $\psi$ is a M-TFW), then we can give an explicit expression of $\psi$ by means of pseudoscaling function in the frequency domain; this is due to $|\operatorname{det} M|=2$. Firstly, we study the pseudoscaling function, generalized low-pass filters, MRA M-TFW, and also give some important characterizations about them. Secondly, we characterize all M-TFW by showing precisely their corresponding dimension functions which are nonnegative integer valued. Finally, we also show that a M-TFW arises from our MRA construction if and only if the dimension of a particular linear space is either zero or one. Our result is a generalization of the construction of TFW from generalized low-pass filters that is introduced in [20,21]. But, it is well known that the situation in higher dimension is more complicated than the situation in one dimension.

Let us now describe the organization of the material as follows. Section 2 presents preliminaries and basic definitions. In Section 3, we study the pseudoscaling function, generalized low-pass filters, and MRA M-TFW, and give some important characterizations about them. In Section 4 , we characterize all M-TFW in $L^{2}\left(\mathbb{R}^{d}\right)$ by dimension function. 


\section{Preliminaries and Basic Definitions}

In this paper, we denote by $D$ the transpose of $M: D=M^{T}$, and $\mathbb{S}=\mathbb{Z}^{d} \backslash D \mathbb{Z}^{d}$, $\varepsilon_{0}$ is a constant vector and $\varepsilon_{0} \in \mathbb{S}$. For $\psi \in L^{2}\left(\mathbb{R}^{d}\right)$, and $k \in \mathbb{Z}^{d}$, we denote

$$
\psi_{j, k}:=2^{j / 2} \psi\left(M^{j} x-k\right) .
$$

Let us recall the concept of frame.

Given a countable index set $I$, a collection $\left\{f_{i}: i \in I\right\}$ in a Hilbert space $\mathbb{H}$ is called a frame in $\mathbb{H}$ if there exist two constants $A, B>0$ such that

$$
A\|f\|_{\mathbb{H}}^{2} \leq \sum_{i \in I}\left|\left\langle f, f_{i}\right\rangle\right|^{2} \leq B\|f\|_{\mathbb{H}}^{2} \quad \forall f \in \mathbb{H} .
$$

If we can choose $A=B$ in (2.2), then $\left\{f_{i}: i \in I\right\}$ is called a tight frame.

Now, we give some basic definitions which will be used in this paper. In fact, they are some generalizations of the notations in $[20,21]$.

Definition 2.1. A function $\psi \in L^{2}\left(\mathbb{R}^{d}\right)$ is a tight frame wavelet with dilation matrix $M$ (briefly: M-TFW) if and only if the system $\left\{\psi_{j, k}, j \in \mathbb{Z}, k \in \mathbb{Z}^{d}\right\}$ is a tight frame (with $A=B=1$ ) for $L^{2}\left(\mathbb{R}^{d}\right)$.

Definition 2.1 implies that

$$
\|f\|_{2}^{2}=\sum_{j \in \mathbb{Z}} \sum_{k \in \mathbb{Z}^{d}}\left|\left\langle f, \psi_{j, k}\right\rangle\right|^{2}, \quad \forall f \in L^{2}\left(\mathbb{R}^{d}\right) .
$$

It is clearly that (2.3) is equivalent to

$$
f=\sum_{j \in \mathbb{Z}} \sum_{k \in \mathbb{Z}^{d}}\left\langle f, \psi_{j, k}\right\rangle \psi_{j, k}
$$

for all $\mathrm{f} \in L^{2}\left(\mathbb{R}^{d}\right)$, where the sum converges unconditionally in $L^{2}\left(\mathbb{R}^{d}\right)$.

Bownik gave a deeper result as follows.

Proposition 2.2 (see [8, Theorem 4.2]). $\psi \in L^{2}\left(\mathbb{R}^{d}\right)$ is a M-TFW if and only if

$$
\begin{gathered}
\sum_{j \in \mathbb{Z}}\left|\widehat{\psi}\left(D^{j} \xi\right)\right|^{2}=1, \quad \text { a.e. } \xi \in \mathbb{R}^{d}, \\
\rho_{k}(\xi)=\sum_{j=0}^{+\infty} \widehat{\psi}\left(D^{j} \xi\right) \overline{\hat{\psi}\left(D^{j}(\xi+2 \pi k)\right)}=0, \quad \text { a.e. } \xi \in \mathbb{R}^{d}, k \in \mathbb{S} .
\end{gathered}
$$


A M-TFW is said to be semi-orthogonal if and only if $\psi_{j, k}$ is orthogonal to $\psi_{\ell, m}$ whenever $j \neq \ell$ (for all $k, m \in \mathbb{Z}^{d}$ ). This is equivalent to the orthogonality of the subspaces $W_{j}$ and $W_{\ell}$ if $j \neq \ell$, where

$$
W_{j}=\overline{\operatorname{span}\left\{\psi_{j, k}: k \in \mathbb{Z}^{d}\right\}}
$$

as $j$ ranges throughout $\mathbb{Z}$.

Proposition 2.3 (see [3, Chapter 7]). Suppose $\psi \in L^{2}\left(\mathbb{R}^{d}\right)$ is a semi-orthogonal M-TFW, then, for each $j \geqslant 1$,

$$
\sum_{k \in \mathbb{Z}^{d}} \widehat{\psi}\left(D^{j}(\xi+2 \pi k)\right) \overline{\widehat{\psi}(\xi+2 \pi k)}=0, \quad \text { a.e. } \xi \in \mathbb{R}^{d}
$$

Definition 2.4. A measurable $2 \pi-\mathbb{Z}^{d}$ periodic function $H$ on $\mathbb{R}^{d}$ is a generalized filter with dilation matrix $M$ (briefly: M-GF) if it satisfies

$$
|H(\xi)|^{2}+\left|H\left(\xi+2 \pi D^{-1} \varepsilon_{0}\right)\right|^{2}=1, \quad \text { a.e. } \xi \in \mathbb{R}^{d}
$$

As what was done in $[20,21]$, we will denote by $\widetilde{F}$ the set of generalized filters with dilation matrix $M$ and let $\widetilde{F}^{+}=\{H \in \widetilde{F}: H \geqslant 0\}$. Observe that $H \in \widetilde{F} \Rightarrow|H| \in \widetilde{F}^{+}$.

Definition 2.5. A function $\varphi \in L^{2}\left(\mathbb{R}^{d}\right)$ is called a pseudoscaling function with dilation matrix $M$ (briefly: M-PSF) if there exists a generalized filter $H \in \widetilde{F}$ such that

$$
\widehat{\varphi}(D \xi)=H(\xi) \widehat{\varphi}(\xi), \quad \text { a.e. } \xi \in \mathbb{R}^{d} .
$$

Remark 2.6. Notice that $H$ is not uniquely determined by the M-PSF $\varphi$. Therefore, we will denote by $\widetilde{F}_{\varphi}$ the set of all $H \in \widetilde{F}$ such that $H$ satisfies (2.10) for $\varphi$. For example, if $\varphi=0$, then $\widetilde{F}_{\varphi}=\widetilde{F}$. If $\varphi$ is a scaling function of MRA wavelet, then $\widetilde{F}_{\varphi}$ is a singleton. On the other hand, if $\varphi$ is an M-PSF, then $|\widehat{\varphi}|^{\vee}$ is also an M-PSF, and if $H \in \widetilde{F}$, then $|H| \in \widetilde{F}_{|\hat{\varphi}|^{v}}$.

Definition 2.7. For $H \in \widetilde{F}$, let

$$
\begin{gathered}
N_{0}(|H|)=\left\{\xi \in \mathbb{R}^{d}: \lim _{j \rightarrow+\infty} \widehat{\varphi}_{|H|}\left(D^{-j} \xi\right)=0\right\}, \\
\widehat{\varphi}_{|H|}(\xi)=\prod_{j=1}^{\infty}\left|H\left(D^{-j} \xi\right)\right| .
\end{gathered}
$$

We say that $H \in \tilde{F}$ is a generalized low-pass filter with dilation matrix M(briefly: M-GLPF) if $\left|N_{0}(|H|)\right|=0$.

Now, we give the definition of MRA M-TFW. 
Definition 2.8. A M-TFW $\psi$ is an MRA M-TFW if there exists a M-PSF $\varphi$ and $H \in \widetilde{F}_{\varphi}$ such that

$$
\widehat{\psi}(D \xi)=e^{i \varepsilon_{0} \cdot \xi} \rho(D \xi) \overline{H\left(\xi+2 \pi D^{-1} \varepsilon_{0}\right)} \widehat{\varphi}(\xi), \quad \text { a.e. } \xi \in \mathbb{R}^{d},
$$

where $\rho(\xi)$ is a measurable $2 \pi-\mathbb{Z}^{d}$ periodic function and $|\rho(\xi)|=1$.

In Section 3, we will prove that if $\psi$ is an MRA M-TFW, then $H$ has to be more than just a generalized filter; $H$ has to be a M-GLPF.

\section{The Characterizations of M-PSF, M-GF, and MRA M-TFW}

The main purpose of this section is to study the M-PSF, the M-GF, and the MRA M-TFW in $L^{2}\left(\mathbb{R}^{d}\right)$. We will give some important characterizations about them.

Lemma 3.1. Suppose $\varphi$ is a M-PSF and $H \in \widetilde{F}_{\varphi}$. If

$$
\lim _{j \rightarrow+\infty}\left|\widehat{\varphi}\left(D^{-j} \xi\right)\right|=1, \quad \text { a.e. } \xi
$$

then,

$$
|\widehat{\varphi}(\xi)|=\left|\prod_{j=1}^{\infty} H\left(D^{-j} \xi\right)\right|, \quad \text { a.e. } \xi,
$$

and $\left|N_{0}(|H|)\right|=0$.

Proof. By (2.10), we have

$$
|\widehat{\varphi}(\xi)|=\left|\prod_{j=1}^{n} H\left(D^{-j} \xi\right)\right|\left|\widehat{\varphi}\left(D^{-n} \xi\right)\right|, \quad \text { a.e. } \xi \in \mathbb{R}^{d}
$$

Using (3.1), we obtain that $|\widehat{\varphi}(\xi)|=\widehat{\varphi}_{|H|}$ and, therefore (3.2) and $\left|N_{0}(|H|)\right|=0$ are clearly satisfied.

Lemma 3.2 (see [25, Lemma 3.2]). If $f \in L^{1}\left(\mathbb{R}^{d}\right)$, then, for a.e. $\omega \in \mathbb{R}^{d}, \lim _{j \rightarrow+\infty}\left|f\left(D^{j} \omega\right)\right|=0$.

Proof. As the proof is simple, we omit it.

Theorem 3.3. Suppose that $\psi$ is an MRA M-TFW and $\varphi$ is a M-PSF satisfying (2.12). Then $H$ defined by (2.12) is a generalized low-pass filter. 
Proof. Suppose that $\psi$ is an MRA M-TFW, by (2.5), (2.9) and (2.12), we can obtain

$$
\begin{aligned}
1=\sum_{j \in \mathbb{Z}}\left|\widehat{\psi}\left(D^{j} \xi\right)\right|^{2} & =\sum_{j \in \mathbb{Z}}\left|H\left(D^{j-1} \xi+2 \pi D^{-1} \varepsilon_{0}\right)\right|^{2}\left|\widehat{\varphi}\left(D^{j-1} \xi\right)\right|^{2} \\
& =\lim _{n \rightarrow+\infty} \sum_{j=-n}^{n}\left|H\left(D^{j-1} \xi+2 \pi D^{-1} \varepsilon_{0}\right)\right|^{2}\left|\widehat{\varphi}\left(D^{j-1} \xi\right)\right|^{2} \\
& =\lim _{n \rightarrow+\infty} \sum_{j=-n}^{n}\left\{1-\left|H\left(D^{j-1} \xi\right)\right|^{2}\right\}\left|\widehat{\varphi}\left(D^{j-1} \xi\right)\right|^{2} \\
& =\lim _{n \rightarrow+\infty}\left\{\left|\widehat{\varphi}\left(D^{-n-1} \xi\right)\right|^{2}-\left|\widehat{\varphi}\left(D^{n-1} \xi\right)\right|^{2}\right\} .
\end{aligned}
$$

Since $\varphi \in L^{2}\left(\mathbb{R}^{d}\right)$, Lemma 3.2 implies $\lim _{n \rightarrow+\infty}\left|\widehat{\varphi}\left(D^{n} \xi\right)\right|^{2}=0$ for a.e. $\xi \in L^{2}\left(\mathbb{R}^{d}\right)$. This shows that for a.e. $\xi \in L^{2}\left(\mathbb{R}^{d}\right), \lim _{n \rightarrow+\infty}\left|\widehat{\varphi}\left(D^{-n} \xi\right)\right|^{2}=1$, thus, by Lemma $3.1, H$ is a generalized low-pass filter.

Suppose that $\psi$ is a M-TFW, let

$$
\begin{gathered}
\sigma_{\psi}(\xi)=\sum_{k \in \mathbb{Z}^{d}}|\widehat{\psi}(\xi+2 \pi k)|^{2}, \\
\operatorname{Dim}_{\psi}(\xi)=\sum_{j=1}^{\infty} \sum_{k \in \mathbb{Z}^{d}}\left|\widehat{\psi}\left(D^{j}(\xi+2 \pi k)\right)\right|^{2} .
\end{gathered}
$$

We say that $\operatorname{Dim}_{\psi}(\xi)$ is the dimension function of M-TFW.

Lemma 3.4. Suppose that $\psi$ is an MRA M-TFW, the corresponding M-PSF of the $\psi$ is $\varphi$, then

$$
|\widehat{\varphi}(\xi)|^{2}=\sum_{j=1}^{\infty}\left|\widehat{\psi}\left(D^{j} \xi\right)\right|^{2}, \quad \text { a.e. } \xi \in \mathbb{R}^{d} \text {. }
$$

Proof. By Definition 2.8, there exists a M-GF $H \in \widetilde{F}$ such that

$$
\widehat{\varphi}(D \xi)=H(\xi) \widehat{\varphi}(\xi), \quad \widehat{\varphi}(D \xi)=e^{i \varepsilon_{0} \cdot \xi} \rho(D \xi) \overline{H\left(\xi+2 \pi D^{-1} \varepsilon_{0}\right)} \widehat{\varphi}(\xi)=H_{1}(\xi) \widehat{\varphi}(\xi)
$$

we obtain that

$$
|\widehat{\varphi}(\xi)|^{2}=|H(\xi)|^{2}|\widehat{\varphi}(\xi)|^{2}+\left|H_{1}(\xi)\right|^{2}|\widehat{\varphi}(\xi)|^{2}=|\widehat{\varphi}(D \xi)|^{2}+|\widehat{\psi}(D \xi)|^{2}
$$


Thus

$$
\begin{aligned}
|\widehat{\varphi}(\xi)|^{2} & =|\widehat{\varphi}(D \xi)|^{2}+|\widehat{\psi}(D \xi)|^{2} \\
& =\left|\widehat{\varphi}\left(D^{2} \xi\right)\right|^{2}+\left|\widehat{\psi}\left(D^{2} \xi\right)\right|^{2}+|\widehat{\psi}(D \xi)|^{2} \\
& =\cdots=\left|\widehat{\varphi}\left(D^{N} \xi\right)\right|^{2}+\left|\widehat{\psi}\left(D^{N} \xi\right)\right|^{2}+\cdots+|\widehat{\psi}(D \xi)|^{2} \\
& =\left|\widehat{\varphi}\left(D^{N} \xi\right)\right|^{2}+\sum_{j=1}^{N}\left|\widehat{\psi}\left(D^{j} \xi\right)\right|^{2} .
\end{aligned}
$$

Notice that $|\widehat{\varphi}(\xi)| \leqslant 1$, hence $\left\{\sum_{j=1}^{N}\left|\widehat{\psi}\left(D^{j} \xi\right)\right|^{2}\right\}_{N}$ is an increased sequence and bounded with 1 . Moreover,

$$
\int_{\mathbb{R}^{d}}\left|\widehat{\varphi}\left(D^{N} \xi\right)\right|^{2} d \xi=\frac{1}{2^{N}} \int_{\mathbb{R}^{d}}|\widehat{\varphi}(\xi)|^{2} d \xi
$$

Therefore, by Fatou lemma,

$$
\begin{aligned}
\int_{\mathbb{R}^{d}} \lim _{N \rightarrow \infty}\left|\widehat{\varphi}\left(D^{N} \xi\right)\right|^{2} d \xi & \leqslant \lim _{N \rightarrow \infty} \int_{\mathbb{R}^{d}}\left|\widehat{\varphi}\left(D^{N} \xi\right)\right|^{2} d \xi \\
& =\lim _{N \rightarrow \infty} \frac{1}{2^{N}} \int_{\mathbb{R}^{d}}|\widehat{\varphi}(\xi)|^{2} d \xi \\
& =0 .
\end{aligned}
$$

So $\lim _{N \rightarrow \infty}\left|\widehat{\varphi}\left(D^{N} \xi\right)\right|^{2}=0$, consequently, $|\widehat{\varphi}(\xi)|^{2}=\sum_{j=1}^{\infty}\left|\widehat{\psi}\left(D^{j} \xi\right)\right|^{2}$, a.e. $\xi \in \mathbb{R}^{d}$.

Lemma 3.5. Suppose that $\psi$ is an MRA M-TFW, then

$$
\operatorname{Dim}_{\psi}(D \xi)+\sigma_{\psi}(D \xi)=\operatorname{Dim}_{\psi}(\xi)+\operatorname{Dim}_{\psi}\left(\xi+2 \pi D^{-1} \varepsilon_{0}\right)
$$

Furthermore, if $\psi$ is a M-TFW, then (3.12) is also valid.

Proof. We only prove the case of $\psi$ is an MRA M-TFW. By Definition 2.8, there exists an M-GF $H \in \widetilde{F}$ such that

$$
\widehat{\varphi}(D \xi)=H(\xi) \widehat{\varphi}(\xi), \quad \widehat{\psi}(D \xi)=e^{i \varepsilon_{0} \cdot \xi} \rho(D \xi) \overline{H\left(\xi+2 \pi D^{-1} \varepsilon_{0}\right)} \widehat{\varphi}(\xi)=H_{1}(\xi) \widehat{\varphi}(\xi) .
$$


By Lemma 3.4, we can obtain

$$
\operatorname{Dim}_{\psi}(\xi)=\sum_{j=1}^{\infty} \sum_{k \in \mathbb{Z}^{d}}\left|\widehat{\psi}\left(D^{j}(\xi+2 \pi k)\right)\right|^{2}=\sum_{k \in \mathbb{Z}^{d}} \sum_{j=1}^{\infty}\left|\widehat{\psi}\left(D^{j}(\xi+2 \pi k)\right)\right|^{2}=\sum_{k \in \mathbb{Z}^{d}}|\widehat{\varphi}(\xi+2 \pi k)|^{2}
$$

Thus,

$$
\begin{aligned}
\operatorname{Dim}_{\psi}(D \xi)= & \sum_{k \in \mathbb{Z}^{d}}|\widehat{\varphi}(D \xi+2 \pi k)|^{2} \\
= & \sum_{k \in D \mathbb{Z}^{d}}|\widehat{\varphi}(D \xi+2 \pi k)|^{2}+\sum_{k \in \mathbb{S}}|\widehat{\varphi}(D \xi+2 \pi k)|^{2} \\
= & \sum_{k \in \mathbb{Z}^{d}}|\widehat{\varphi}(D(\xi+2 \pi k))|^{2}+\sum_{k \in \mathbb{Z}^{d}}\left|\widehat{\varphi}\left(D\left(\xi+2 \pi k+2 \pi D^{-1} \varepsilon_{0}\right)\right)\right|^{2} \\
= & \sum_{k \in \mathbb{Z}^{d}}|H(\xi+2 \pi k)|^{2}|\widehat{\varphi}(\xi+2 \pi k)|^{2} \\
& +\sum_{k \in \mathbb{Z}^{d}}\left|H\left(\xi+2 \pi k+2 \pi D^{-1} \varepsilon_{0}\right)\right|^{2}\left|\widehat{\varphi}\left(\xi+2 \pi k+2 \pi D^{-1} \varepsilon_{0}\right)\right|^{2} \\
= & |H(\xi)|^{2} \sum_{k \in \mathbb{Z}^{d}}|\widehat{\varphi}(\xi+2 \pi k)|^{2}+\left|H\left(\xi+2 \pi D^{-1} \varepsilon_{0}\right)\right|^{2}\left|\sum_{k \in \mathbb{Z}^{d}} \hat{\varphi}\left(\xi+2 \pi k+2 \pi D^{-1} \varepsilon_{0}\right)\right|^{2} \\
= & |H(\xi)|^{2} \operatorname{Dim}_{\psi}(\xi)+\left|H\left(\xi+2 \pi D^{-1} \varepsilon_{0}\right)\right|^{2} \operatorname{Dim}_{\psi}\left(\xi+2 \pi D^{-1} \varepsilon_{0}\right) .
\end{aligned}
$$

Analogously, we have

$$
\sigma_{\psi}(D \xi)=\left|H_{1}(\xi)\right|^{2} \operatorname{Dim}_{\psi}(\xi)+\left|H_{1}\left(\xi+2 \pi D^{-1} \varepsilon_{0}\right)\right|^{2} \operatorname{Dim}_{\psi}\left(\xi+2 \pi D^{-1} \varepsilon_{0}\right) .
$$

Thus,

$$
\operatorname{Dim}_{\psi}(D \xi)+\sigma_{\psi}(D \xi)=\operatorname{Dim}_{\psi}(\xi)+\operatorname{Dim}_{\psi}\left(\xi+2 \pi D^{-1} \varepsilon_{0}\right)
$$

Theorem 3.6. Suppose that $\psi$ is an MRA M-TFW, the corresponding M-PSF of the $\psi$ is $\varphi$, then

$$
0 \leqslant \operatorname{Dim}_{\psi}(\xi) \leqslant 1, \text { a.e. } \xi \in \mathbb{R}^{d}
$$


Proof. Let $H \in \widetilde{F}_{\varphi}$, and it is the corresponding M-GLPF of the $\psi$. Let

$$
\widehat{\varphi}_{n}(\xi)= \begin{cases}\prod_{j=1}^{n} H\left(D^{-j} \xi\right) \chi_{2^{n / d} \mathbb{T}^{d}}(\xi), & n=q d, q \in \mathbb{Z}, \\ \prod_{j=1}^{n+1} H\left(D^{-j} \xi\right) \chi_{2^{(n+1) / d} \mathbb{T}^{d}(\xi),} & n=q d-1, q \in \mathbb{Z}, \\ \vdots & \\ \prod_{j=1}^{n+(d-1)} H\left(D^{-j} \xi\right) \chi_{2^{(n+d-1) / d} \mathbb{T}^{d}}(\xi), & n=q d-(d-1), q \in \mathbb{Z},\end{cases}
$$

where $\Gamma$ denotes all the vertices of cube $[0,1]^{d}, \mathbb{T}=[0,2 \pi]$. It is clearly that $\lim _{n \rightarrow \infty}\left|\widehat{\varphi}_{n}(\xi)\right|=$ $|\widehat{\varphi}(\xi)|$. Now, we need to prove that $\left\{\varphi_{n}(\cdot-k)\right\}_{k \in \mathbb{Z}^{d}}$ is an orthonormal system for $L^{2}\left(\mathbb{R}^{d}\right)$. Denote

$$
I_{n}=\int_{\mathbb{R}^{d}} \varphi_{n}(x) \overline{\varphi_{n}(x-k)} d x .
$$

So, if $n=q d, q \in \mathbb{Z}$, then

$$
\begin{aligned}
I_{n} & =\int_{\mathbb{R}^{d}} \varphi_{n}(x) \overline{\varphi_{n}(x-k)} d x=\frac{1}{(2 \pi)^{d}} \int_{[0,2 q 2 \pi]^{d}} \prod_{j=1}^{n}\left|H\left(D^{-j} \xi\right)\right|^{2} e^{i k \xi} d \xi \\
& =\frac{1}{(2 \pi)^{d}} \int_{[0,2 q 2 \pi]^{d}} \prod_{j=1}^{(q-1) d}\left|H\left(D^{-j} \xi\right)\right|^{2} e^{i k \xi}\left\{\prod_{\ell=0}^{d-1}\left|H\left(D^{-q d+\ell} \xi\right)\right|^{2}\right\} d \xi \\
& =\frac{1}{(2 \pi)^{d}} \int_{[0,2 q-12 \pi]^{d}} \prod_{j=1}^{(q-1) d}\left|H\left(D^{-j} \xi\right)\right|^{2} e^{i k \xi} \times\left\{\sum_{\omega \in \Gamma} \prod_{\ell=0}^{d-1}\left|H\left(D^{-q d+\ell}\left(\xi+2^{q} \pi \omega\right)\right)\right|^{2}\right\} d \xi \\
& =\frac{1}{(2 \pi)^{d}} \int_{[0,2 q-12 \pi]^{d}} \prod_{j=1}^{(q-1) d}\left|H\left(D^{-j} \xi\right)\right| e^{i k \xi} \\
& =I_{(q-1) d}=\cdots=I_{d} .
\end{aligned}
$$

However,

$$
\begin{aligned}
I_{d} & =\frac{1}{(2 \pi)^{d}} \int_{[0,4 \pi]^{d}} \prod_{j=1}^{d}\left|H\left(D^{-j} \xi\right)\right|^{2} e^{i k \xi} d \xi \\
& =\frac{1}{(2 \pi)^{d}} \int_{[0,2 \pi]^{d}} e^{i k \xi} \times\left\{\sum_{\omega \in \Gamma \ell=1}^{d}\left|H\left(D^{-\ell}(\xi+2 \pi \omega)\right)\right|^{2}\right\} d \xi \\
& =\frac{1}{(2 \pi)^{d}} \int_{[0,2 \pi]^{d}} e^{i k \xi}=\delta_{k, 0} .
\end{aligned}
$$


Thus we deduce from (3.19) and the above equation that if $n=q d, q \in \mathbb{Z}$, then

$$
\left\langle\varphi_{n}(\cdot), \varphi_{n}(\cdot-k)\right\rangle=\delta_{k, 0} .
$$

Similarly, by (3.19), (3.23) also holds if $n=q d-1$, or $n=q d-2, \ldots$, or $n=q d-(d-1)$. Therefore, for a.e. $\xi \in \mathbb{R}^{d}$

$$
\sum_{k \in \mathbb{Z}^{d}}\left|\widehat{\varphi}_{n}(\xi+2 \pi k)\right|^{2}=1, \quad \forall n \in \mathbb{N} .
$$

By Fatou lemma,

$$
\operatorname{Dim}_{\psi}(\xi)=\sum_{k \in \mathbb{Z}^{d}}|\widehat{\varphi}(\xi+2 \pi k)|^{2}=\sum_{k \in \mathbb{Z}^{d}} \lim _{n \rightarrow \infty}\left|\widehat{\varphi}_{n}(\xi+2 \pi k)\right|^{2} \leqslant \lim _{n \rightarrow \infty} \sum_{k \in \mathbb{Z}^{d}}\left|\widehat{\varphi}_{n}(\xi+2 \pi k)\right|^{2}=1 .
$$

Recall that the MRA TFW are precisely those $\psi \in L^{2}(\mathbb{R})$ that can be constructed from a generalized low-pass filter as described in $[20,21]$. What are the properties of $\sigma_{\psi}$ when $\psi \in L^{2}\left(\mathbb{R}^{d}\right)$ is a M-TFW? We will see that the answers to this question are important for determining the properties of $\operatorname{Dim}_{\psi}$.

For $\psi \in L^{2}\left(\mathbb{R}^{d}\right)$ (not necessarily a M-TFW), let us consider the principal shift-invariant space $W(\psi) \equiv \overline{\operatorname{span}\left\{\psi(\cdot-k): k \in \mathbb{Z}^{d}\right\}}$. When $\psi$ is an M-TFW, then the space $W_{0}$ we defined in (2.7) is the space $W(\psi)$. We will be interested in examining what type of spanning set for $W_{0}$ is $\{\psi(\cdot-k)\}=\left\{\psi_{0 k}\right\}, k \in \mathbb{Z}^{d}$. If $\psi$ is an orthonormal wavelet, then $\{\psi(\cdot-k)\}$ is an orthonormal basis for $W_{0}$. What will happen if $\psi \in L^{2}\left(\mathbb{R}^{d}\right)$ is a M-TFW?

It is not hard to see that we can find $\varphi \in W(\psi)$ such that $\left\{\varphi(\cdot-k), k \in \mathbb{Z}^{d}\right\}$ is a tight frame (with constant 1) for $W(\psi)$. Indeed, let $s(\xi)=1 / \sqrt{\sigma_{\psi}(\xi)}$ if $\xi \in U_{\psi}$ and $s(\xi)=0$ if $\xi \notin U_{\psi}$, where $U_{\psi}=U=\left\{\xi \in \mathbb{T}, \sigma_{\psi}>0\right\}$. Straight forward calculations show that $\varphi$ defined by $\widehat{\varphi}(\xi)=s(\xi) \widehat{\psi}(\xi)$ provides us with the desired function. Moreover,

$$
\sigma_{\varphi}=\chi U_{\psi}(\xi)
$$

and $U_{\varphi}=U_{\psi}$. This result can be extended to the following known result.

Lemma 3.7 (see [5, Theorem 7.2.3]). $\{\psi(\cdot-k)\}, k \in \mathbb{Z}^{d}$, is a tight frame with constant $A, B$ for $W(\psi)$ if and only if

$$
A_{\chi U} \leqslant \sigma_{\psi} \leqslant B \chi U
$$

When $\psi$ is a M-TFW, for the space $W_{0}$, one has the following results.

Lemma 3.8 (see [21, Theorem 2.7]). Suppose that $\psi$ is a M-TFW. The followings are equivalent:

(a) $\{\psi(\cdot-k)\}_{k \in \mathbb{Z}^{d}}$ is a tight frame (of constant 1) for $W_{0}$ (i.e., $\psi$ is a $W_{0}-T F$ ),

(b) $\|\psi\|_{2}^{2}=\sum_{k \in \mathbb{Z}^{d}}\left|\left\langle\psi, \psi_{0 k}\right\rangle\right|^{2}$,

(c) $\psi$ is semi-orthogonal,

(d) $\sigma_{\psi}=\chi_{U}$ a.e. on $\mathbb{R}^{d}$. 
Proof. It is clear that (a) implies (b), this is (2.3) with $f=\psi, H=W_{0}$. Since

$$
\|\psi\|_{2}^{2}=\sum_{k \in \mathbb{Z}^{d}}|\langle\psi, \psi 0 k\rangle|^{2}
$$

and $\left\{\psi_{j k}\right\}$ is a tight frame, (b) implies $\left\langle\psi_{,} \psi_{j k}\right\rangle=0$ whenever $j \neq 0$. This clearly implies the semiorthogonality. Thus, (b) implies (c). If we assume (c), so that $\left\langle f, \psi_{j k}\right\rangle=0$ whenever $f \in W_{0}$ and $j \neq 0$, an application of (2.3) gives us

$$
\|f\|_{2}^{2}=\sum_{j \in \mathbb{Z}} \sum_{k \in \mathbb{Z}^{d}}\left|\left\langle f, \psi_{j k}\right\rangle\right|^{2}=\sum_{k \in \mathbb{Z}^{d}}\left|\left\langle f, \psi_{0 k}\right\rangle\right|^{2}
$$

and we see that (c) implies (a).

Lemma 3.7 with $A=B=1$ tells us that (a) and (d) are equivalent.

Although the proof of Lemma 3.8 is rather simple, the result is not obvious. Some aspects of this lemma are counter intuitive: the properties (a), (b), and (d) are tied to the inner structure of $W(\psi)=W_{0}$. On the other hand, (c) provides information about the relationship between $W_{0}$ and the other space $W_{j}, j \neq 0$. The assumption that $\psi$ is an M-TFW is very important. If we do not assume this to be the case, (a) and (d) are still equivalent. However, (b) is not equivalent to (d) (see [21]).

Now, we need to make some facts clear. For Theorem 3.3, Lemma 3.4, and Lemma 3.8, the assumption of $M^{d}=2 I_{d}$ can be weakened into $|\operatorname{det} M|=2$, and the assumption of integer-valued dilation matrix can be weakened into noninteger-valued dilation matrix, Theorem 3.3, Lemma 3.4, and Lemma 3.8 still hold; their proofs work more or less in an unchanged form from the original ones. On the other hand, for Lemma 3.5 and Theorem 3.6, the assumption of integer-valued dilation matrix cannot be weakened into noninteger-valued dilation matrix, because we can obtain $D \mathbb{Z}^{d} \subset \mathbb{Z}^{d}$ and $\mathbb{Z}^{d}=D \mathbb{Z}^{d}+\mathbb{Z}^{d} \backslash D \mathbb{Z}^{d}$ if and only if dilation matrix is integer valued, in fact, during the course of these proofs we always use $\sum_{k \in \mathbb{Z}^{d}}=\sum_{k \in D \mathbb{Z}^{d}}+\sum_{\mathbb{Z}^{d} \backslash D \mathbb{Z}^{d}}$.

\section{Dimension Function of M-TFW}

The dimension function of a multivariate orthonormal wavelet $\psi$ is integer value; moreover, unless $\psi$ is an MRA wavelet, it attains each of the integer values in the interval $[0, M]$, where $M$ is the supremum of $\operatorname{Dim}_{\psi}$, on sets of positive measure (see $[8,21]$ ). We will investigate the properties of the dimension function for M-TFW.

Theorem 4.1. Suppose that $\psi$ is a M-TFW. Then $\psi$ is semi-orthogonal if and only if $\operatorname{Dim}_{\psi}$ is integer valued a.e.

Proof. First, let us prove the sufficiency. Suppose that $\operatorname{Dim}_{\psi}$ is integer valued a.e. From Lemma 3.5, we see that $\sigma_{\psi}$ must also be integer valued a.e. By (3.18) and $\sigma_{\psi}(\xi) \leqslant 1$ a.e., we have $\sigma_{\psi}(\xi)=X U_{\psi}(\xi)$ a.e. By Lemma 3.8, part (c) and (d), we conclude that $\psi$ is semiorthogonal. 
Now, we prove the necessity. Let us assume that $\psi$ is semi-orthogonal, denote

$$
G_{n}(\xi)=\sum_{j=1}^{\infty} \widehat{\psi}\left(D^{j} \xi\right) \sum_{k \in \mathbb{Z}^{d}} \widehat{\psi}\left(D^{n}(\xi+2 \pi k)\right) \overline{\widehat{\psi}\left(D^{j}(\xi+2 \pi k)\right)}, \quad n \geqslant 1 .
$$

Apply Schwart's inequality to the sum in (4.1) with respect to $k$, we obtain

$$
\begin{aligned}
\sum_{j=1}^{\infty} \mid \widehat{\psi} & \left(D^{j} \xi\right)\left|\sum_{k \in \mathbb{Z}^{d}}\right| \widehat{\psi}\left(D^{n}(\xi+2 \pi k)\right)|\cdot| \widehat{\psi}\left(D^{j}(\xi+2 \pi k)\right) \mid \\
& \leqslant \sum_{j=1}^{\infty}\left|\widehat{\psi}\left(D^{j} \xi\right)\right|\left(\sum_{k \in \mathbb{Z}^{d}}\left|\widehat{\psi}\left(D^{n}(\xi+2 \pi k)\right)\right|^{2}\right)^{1 / 2}\left(\sum_{k \in \mathbb{Z}^{d}}\left|\widehat{\psi}\left(D^{j}(\xi+2 \pi k)\right)\right|^{2}\right)^{1 / 2} \\
& \leqslant \sum_{j=1}^{\infty}\left|\widehat{\psi}\left(D^{j} \xi\right)\right|\left(\sum_{k \in \mathbb{Z}^{d}}\left|\widehat{\psi}\left(D^{j}(\xi+2 \pi k)\right)\right|^{2}\right)^{1 / 2}\left(\sigma_{\psi}\left(D^{n} \xi\right)\right)^{1 / 2}
\end{aligned}
$$

Using the fact that $\sigma_{\psi}\left(D^{n} \xi\right) \leqslant 1$ a.e. and appling Schwart's inequality to the sum over $j$, we obtain that

$$
\left(\sum_{j=1}^{\infty}\left|\widehat{\psi}\left(D^{j} \xi\right)\right|^{2}\right)^{1 / 2}\left(\sum_{j=1}^{\infty} \sum_{k \in \mathbb{Z}^{d}}\left|\widehat{\psi}\left(D^{j}(\xi+2 \pi k)\right)\right|^{2}\right)^{1 / 2} \leqslant \sqrt{\operatorname{Dim}_{\psi}(\xi)}
$$

Therefore, the sum in (4.1) is absolutely convergent. The fact allows us to interchange the sums in the expression $G_{n}(\xi)$. By Proposition 2.3, we have

$$
\begin{aligned}
G_{n}(\xi)= & \sum_{k \in \mathbb{Z}^{d}} \widehat{\psi}\left(D^{n}(\xi+2 \pi k)\right) \sum_{j=1}^{\infty} \widehat{\psi}\left(D^{j} \xi\right) \overline{\widehat{\psi}\left(D^{j}(\xi+2 \pi k)\right)} \\
& +\widehat{\psi}(\xi) \sum_{k \in \mathbb{Z}^{d}} \widehat{\psi}\left(D^{n}(\xi+2 \pi k)\right) \overline{\widehat{\psi}(\xi+2 \pi k)} \\
= & \sum_{k \in \mathbb{Z}^{d}} \widehat{\psi}\left(D^{n}(\xi+2 \pi k)\right) \sum_{j=0}^{\infty} \widehat{\psi}\left(D^{j} \xi\right) \overline{\widehat{\psi}\left(D^{j}(\xi+2 \pi k)\right)} \\
= & \sum_{k \in \mathbb{Z}^{d}} \widehat{\psi}\left(D^{n}(\xi+2 \pi k)\right) \rho_{k}(\xi),
\end{aligned}
$$

where $\varphi_{k}(\xi)$ is defined as in (2.6). Since $\varphi_{k}(\xi)=0$, a.e. $\xi \in \mathbb{R}^{d}, k \in \mathbb{S}$, we obtain

$$
G_{n}(\xi)=\sum_{k \in \mathbb{Z}^{d}} \widehat{\psi}\left(D^{n}(\xi+2 \pi D k)\right) \sum_{j=0}^{\infty} \widehat{\psi}\left(D^{j} \xi\right) \overline{\widehat{\psi}\left(D^{j}(\xi+2 \pi D k)\right)} .
$$


On the other hand,

$$
\begin{aligned}
G_{n+1}\left(D^{-1} \xi\right) & =\sum_{k \in \mathbb{Z}^{d}} \widehat{\psi}\left(D^{n+1}\left(D^{-1} \xi+2 \pi k\right)\right) \sum_{j=1}^{\infty} \widehat{\psi}\left(D^{j} D^{-1} \xi\right) \overline{\widehat{\psi}\left(D^{j}\left(D^{-1} \xi+2 \pi k\right)\right)} \\
& =\sum_{k \in \mathbb{Z}^{d}} \widehat{\psi}\left(D^{n}(\xi+2 \pi D k)\right) \sum_{j=0}^{\infty} \widehat{\psi}\left(D^{j} \xi\right) \overline{\widehat{\psi}\left(D^{j}(\xi+2 \pi D k)\right)} \\
& =G_{n}(\xi) .
\end{aligned}
$$

Therefore, we have shown that $G_{n}(\xi)=G_{n-1}(D \xi)$, and consequently, $G_{n}(\xi)=G_{1}\left(D^{n-1} \xi\right)$ for $n \geqslant 1$. By Propositions 2.2 and 2.3, we have

$$
\begin{aligned}
G_{1}(\xi)= & \sum_{k \in \mathbb{Z}^{d}} \widehat{\psi}(D(\xi+2 \pi k)) \sum_{j=1}^{\infty} \widehat{\psi}\left(D^{j} \xi\right) \overline{\widehat{\psi}\left(D^{j}(\xi+2 \pi k)\right)} \\
= & \sum_{k \in \mathbb{Z}^{d}} \widehat{\psi}(D \xi+2 \pi D k) \sum_{j=0}^{\infty} \widehat{\psi}\left(D^{j} \cdot D \xi\right) \overline{\widehat{\psi}\left(D^{j}(D \xi+2 \pi D k)\right)} \\
= & \sum_{k \in \mathbb{Z}^{d}} \widehat{\psi}(D \xi+2 \pi D k) \sum_{j=0}^{\infty} \widehat{\psi}\left(D^{j} \cdot D \xi\right) \overline{\widehat{\psi}\left(D^{j}(D \xi+2 \pi D k)\right)} \\
& +\sum_{k \in \mathbb{S}} \widehat{\psi}(D \xi+2 \pi k) \sum_{j=0}^{\infty} \widehat{\psi}\left(D^{j} \cdot D \xi\right) \overline{\widehat{\psi}\left(D^{j}(D \xi+2 \pi k)\right)} \\
= & \sum_{k \in \mathbb{Z}^{d}} \widehat{\psi}(D \xi+2 \pi k) \sum_{j=0}^{\infty} \widehat{\psi}\left(D^{j} \cdot D \xi\right) \overline{\widehat{\psi}\left(D^{j}(D \xi+2 \pi k)\right)} \\
= & \sum_{j=0}^{\infty} \widehat{\psi}\left(D^{j} \cdot D \xi\right) \sum_{k \in \mathbb{Z}^{d}} \widehat{\psi}(D \xi+2 \pi k) \overline{\widehat{\psi}\left(D^{j}(D \xi+2 \pi k)\right)} \\
= & \sum_{j=1}^{\infty} \widehat{\psi}\left(D^{j} \cdot D \xi\right) \sum_{k \in \mathbb{Z}^{d}} \widehat{\psi}(D \xi+2 \pi k) \overline{\widehat{\psi}\left(D^{j}(D \xi+2 \pi k)\right)}+\widehat{\psi}(D \xi) \sigma_{\psi}(D \xi) \\
= & \widehat{\psi}(D \xi) \sigma_{\psi}(D \xi) .
\end{aligned}
$$

This shows that

$$
G_{n}(\xi)=\widehat{\psi}\left(D^{n} \xi\right) \sigma_{\psi}\left(D^{n} \xi\right)
$$

Finally, since $\psi$ is a semi-orthogonal M-TFW, $\sigma_{\psi}\left(D^{n} \xi\right)$ is either 0 or 1 a.e.; this and the last fact, which implies that $\widehat{\psi}\left(D^{n} \xi\right)=0$ when $\sigma_{\psi}\left(D^{n} \xi\right)=0$, give us

$$
\widehat{\psi}\left(D^{n} \xi\right)=G_{n}(\xi), \quad \text { a.e., } n \geqslant 1 .
$$


Now, let

$$
\Psi_{j}(\xi)=\left\{\widehat{\psi}\left(D^{j}(\xi+2 k \pi)\right): k \in \mathbb{Z}^{d}\right\}, \quad j \geqslant 1
$$

Using the fact that $\sigma_{\psi}(\xi) \leqslant 1$ once again, we see that $\Psi_{j} \in \ell^{2}\left(\mathbb{Z}^{d}\right)$ for a.e. $\xi \in \mathbb{R}^{d}$. But, from (2.6), (2.7), and (2.8), we see that

$$
\Psi_{j}(\xi)=\sum_{n=1}^{\infty}\left\langle\Psi_{n}(\xi), \Psi_{j}(\xi)\right\rangle_{\ell^{2}\left(\mathbb{Z}^{d}\right)} \Psi_{n}(\xi)
$$

for $j \geqslant 1$.

By the definition of $\operatorname{Dim}_{\psi}$, we have

$$
\operatorname{Dim}_{\psi}(\xi)=\sum_{j=1}^{\infty}\left\|\Psi_{j}(\xi)\right\|_{\ell^{2}\left(\mathbb{Z}^{d}\right)}^{2}
$$

Hence, we conclude that

$$
\operatorname{Dim}_{\psi}(\xi)=\operatorname{dim} \mathbb{F}_{\psi}(\xi) \text {, a.e. } \xi \in \mathbb{R}^{d},
$$

where $\mathbb{F}_{\psi}(\xi)=\overline{\operatorname{span}\left\{\Psi_{j}(\xi): j \geqslant 1\right\}}$; this is a well-defined subspace of $\ell^{2}\left(\mathbb{Z}^{d}\right)$. Obviously, (4.13) implies that $\operatorname{Dim}_{\psi}$ is integer valued a.e. The proof of Theorem 4.1 is completed.

Theorem 4.1 provides us with the following interesting statement.

Corollary 4.2. Suppose that $\psi$ is a M-TFW. Then $\operatorname{Dim}_{\psi}(\xi)=\operatorname{dim} \mathbb{F}_{\psi}(\xi)$, for a.e. $\xi \in \mathbb{R}^{d}$, if and only if $\psi$ is semi-orthogonal.

The dimension function of an orthonormal wavelet which attains value $N>1$ on a set of positive measure, must also attain value $N-1$ on a set of positive measure (for details, see [14]). Equation (3.12) is used there to prove that

$$
\operatorname{Dim}_{\psi}(D \xi) \geqslant \operatorname{Dim}_{\psi}(\xi)-1, \quad \text { a.e. } \xi \text {. }
$$

Fortunately, (4.14) is also valid for semi-orthogonal M-TFWs. More precisely, one has the following result.

Proposition 4.3. Suppose that $\psi$ is a semi-orthogonal M-TFW. Let $N>1$ be an integer. If there exits a set $A \subseteq \mathbb{R}^{d}$ of positive Lebesgue measure, such that $\operatorname{Dim}_{\psi}(\xi) \geqslant N$ for all $\xi \in A$, then there exits a set $B \subseteq \mathbb{R}^{d}$ of positive Lebesgue measure such that $\operatorname{Dim}_{\psi}(\xi)=N-1$ for all $\xi \in B$.

There is another interesting consequence of Theorem 4.1. Notice that a consequence of (2.5) is that for every M-TFW $\psi$, one has

$$
|\widehat{\psi}(\xi)| \leqslant 1, \quad \text { a.e. }
$$


An interesting class consists of those M-TFW for which $|\widehat{\psi}(\xi)|$ attains only values 0 and 1 ; in accordance with the orthonormal wavelet terminology one will call such M-TFW, MSF MTFW. One may expect that MSF M-TFW may or may not be semi-orthogonal. Theorem 4.1, however, implies that they have to lie within the realm of semi-orthogonal M-TFW since the corresponding dimension function must be integer-valued. Let us state this in the form of a corollary (see [21]).

Corollary 4.4. If $\psi$ is an MSF M-TFW, then $\psi$ is semi-orthogonal.

Theorem 4.5. Suppose that $\psi$ is a M-TFW, then, $\psi$ is an MRA M-TFW if and only if $\operatorname{dim} \mathbb{F}_{\psi}(\xi) \in$ $\{0,1\}$, for a.e. $\xi \in \mathbb{R}^{d}$.

Proof of Theorem 4.5. First, we prove the necessity. Suppose that $\psi$ is an MRA M-TFW, thus, it satisfies (2.12) for an appropriate $H, \rho$ and $\varphi$. If $\operatorname{dim} \mathbb{F}_{\psi}(\xi) \geqslant 2$, for some $\xi \in \mathbb{R}^{d}$, then there exist $k, \ell$ such that $\Psi_{k}(\xi)$ and $\Psi_{\ell}(\xi)$ are linearly independent in $\ell^{2}\left(\mathbb{Z}^{d}\right)$. It is then impossible to have a vector $v \in \ell^{2}\left(\mathbb{Z}^{d}\right)$ such that both $\Psi_{k}(\xi)$ and $\Psi_{\ell}(\xi)$ are in the one-dimensional subspace $\operatorname{span}\{v\}$. However, by (2.10) and (2.12), for every $j \geqslant 1$, we have

$$
\begin{aligned}
& \widehat{\psi}\left(D^{j}(\xi+2 \pi k)\right) \\
& =e^{i \varepsilon_{0} \cdot D^{j-1}(\xi+2 \pi k)} \rho\left(D^{j}(\xi+2 \pi k)\right) \overline{H\left(D^{j-1}(\xi+2 \pi k)+2 \pi D^{-1} \varepsilon_{0}\right)} \hat{\varphi}\left(D^{j-1}(\xi+2 \pi k)\right) \\
& =e^{i \varepsilon_{0} \cdot D^{j-1} \xi} \rho\left(D^{j} \xi\right) \overline{H\left(D^{j-1} \xi+2 \pi D^{-1} \varepsilon_{0}\right)} \prod_{n=0}^{j-2} H\left(D^{n} \xi\right) \widehat{\varphi}(\xi+2 \pi k), \quad k \in \mathbb{Z}^{d} .
\end{aligned}
$$

Since $\left\{\widehat{\varphi}(\xi+2 \pi k): k \in \mathbb{Z}^{d}\right\}$ is in $\ell^{2}\left(\mathbb{Z}^{d}\right)$, for a.e. $\xi \in \mathbb{R}^{d}$, by Lemma 3.5 and Theorem 3.6, we conclude that $\operatorname{dim} \mathbb{F}_{\psi}(\xi)$ is at most 1 , for a.e. $\xi \in \mathbb{R}^{d}$.

Now, we will prove the sufficiency. In other words, we have to prove that $\psi$ is an MRA M-TFW, assuming that $\psi$ is an M-TFW such that $\operatorname{dim} \mathbb{F}_{\psi}(\xi)$ is either 0 or 1 , for a.e. $\xi \in \mathbb{R}^{d}$. We noticed that $\mathbb{F}_{\psi}(\xi)$ is always defined for a M-TFW $\psi$ and is a closed subspace of $\ell^{2}\left(\mathbb{Z}^{d}\right)$. Theorem 4.1 implies that, for $j \geqslant 1, \Psi_{j}(\xi) \in \ell^{2}\left(\mathbb{Z}^{d}\right)$, for a.e. $\xi \in \mathbb{R}^{d}$.

Therefore, we need to prove that there exist a generalized filter $H$ and a corresponding pseudoscaling function $\varphi$ such that (2.12) is satisfied, that is, a.e. $\xi \in \mathbb{R}^{d}$

$$
\widehat{\psi}(D \xi)=e^{i \varepsilon_{0} \cdot \xi} \rho(D \xi) \overline{H\left(\xi+2 \pi D^{-1} \varepsilon_{0}\right)} \widehat{\varphi}(\xi) .
$$

Thus, we begin to prove that it is enough to show that there exist a generalized filter $H_{0}$, a corresponding pseudoscaling function $\varphi_{0}$, and a $2 \pi-\mathbb{Z}^{d}$ periodic, unimodular function $\rho$ such that

$$
\widehat{\psi}(D \xi)=e^{i \varepsilon_{0} \cdot \xi} \rho(D \xi) \overline{H_{0}\left(\xi+2 \pi D^{-1} \varepsilon_{0}\right)} \widehat{\varphi_{0}}(\xi)
$$

Let,

$$
\mathcal{Z}:=\left\{\xi \in \mathbb{R}^{d}: \operatorname{Dim}_{\psi}(\xi)=0\right\}
$$


and, for $j \in \mathbb{N}$, we define the set $p_{j}$ to be

$$
p_{j}:=\left\{\xi \in \mathbb{R}^{d}:\left\|\Psi_{j}(\xi)\right\|_{\ell^{2}\left(\mathbb{Z}^{d}\right)} \neq 0,\left\|\Psi_{\ell}(\xi)\right\|_{2}=0 \text { for } 1 \leqslant \ell \leqslant j-1\right\}
$$

It is clearly that $z$ is the set where all vectors $\Psi_{j}(\xi), j \geqslant 1$, are zero (or, equivalently, where $\operatorname{dim} \mathbb{F}_{\psi}(\xi)=0$ ). Moreover, the set $z$ and the $p_{j}, j \geqslant 1$, are $2 \pi$ - $\mathbb{Z}^{d}$ periodic, measurable and they form a partition of $\mathbb{R}^{d}$.

We define $\widehat{\varphi_{0}}$ as follows:

$$
\widehat{\varphi_{0}}(\xi)= \begin{cases}0, & \xi \in \mathcal{Z}, \\ \sqrt{\frac{\operatorname{Dim}_{\psi}(\xi)}{\sum_{k \in \mathbb{Z}^{d}}\left|\widehat{\psi}\left(D^{j}(\xi+2 \pi k)\right)\right|^{2}}} \cdot \widehat{\psi}\left(D^{j} \xi\right), & \xi \in \mathcal{D}_{j} .\end{cases}
$$

Observe that (4.21) makes sense and defines a measurable function $\widehat{\varphi_{0}}: \mathbb{R}^{d} \rightarrow \mathbb{C}$. Furthermore, the function clearly satisfies

$$
\sum_{k \in \mathbb{Z}^{d}}\left|\widehat{\varphi_{0}}(\xi+2 \pi k)\right|^{2}=\operatorname{Dim}_{\psi}(\xi), \quad \text { a.e. }
$$

Using (4.22), we obtain that $\left\|\widehat{\varphi_{0}}\right\|_{2}^{2}=\int_{\mathbb{T}^{d}} \operatorname{Dim}_{\psi}(\xi) d \xi$. It follows that $\widehat{\varphi_{0}} \in L^{2}\left(\mathbb{R}^{d}\right)$. Thus, $\varphi_{0} \in$ $L^{2}\left(\mathbb{R}^{d}\right)$.

Next, we divide the argument into six steps (six lemmas).

Lemma 4.6. For a.e. $\xi \in \mathbb{R}^{d}$, one has

$$
\left|\widehat{\varphi_{0}}(\xi)\right|^{2}=\sum_{j=1}^{\infty}\left|\widehat{\psi}\left(D^{j} \xi\right)\right|^{2}
$$

Proof. If $\xi \in \mathcal{Z}$, then, (4.23) is trivially true. We only need to consider $\xi \in p_{\ell}, \ell \in \mathbb{N}$. Thus, $\Psi_{\ell}(\xi) \neq 0$ and, by assumption, $\operatorname{dim} \mathbb{F}_{\psi}(\xi)=1$. This implies that for every $j \geqslant 1$ there exists a $2 \pi-\mathbb{Z}^{d}$ periodic, measurable function $\lambda_{j}^{\ell}: D_{\ell} \rightarrow \mathbb{C}$, such that, for a.e. $\xi \in D_{\ell}$,

$$
\Psi_{j}(\xi)=\lambda_{j}^{\ell}(\xi) \Psi_{l}(\xi)
$$

Coordinatewise, this means that for every $k \in \mathbb{Z}^{d}$, a.e. $\xi \in D_{\ell}$

$$
\widehat{\psi}\left(D^{j}(\xi+2 \pi k)\right)=\lambda_{j}^{\ell}(\xi) \widehat{\psi}\left(D^{\ell}(\xi+2 \pi k)\right) .
$$

It follows that the right hand side of (4.23) satisfies

$$
\sum_{j=1}^{\infty}\left|\widehat{\psi}\left(D^{j} \xi\right)\right|^{2}=\left[\sum_{j=1}^{\infty}\left|\lambda_{j}^{\ell}(\xi)\right|^{2}\right] \cdot\left|\widehat{\psi}\left(D^{\ell} \xi\right)\right|^{2}
$$


for a.e. $\xi \in D_{\ell}$. Keeping in mind formula (4.21) for $\xi \in D_{\ell},(4.26)$ implies that

$$
\sum_{j=1}^{\infty}\left|\lambda_{j}^{\ell}(\xi)\right|^{2}=\frac{\sum_{j=1}^{\infty}\left|\widehat{\psi}\left(D^{j} \xi\right)\right|^{2}}{\left|\widehat{\psi}\left(D^{\ell} \xi\right)\right|^{2}},
$$

for a.e. $\xi \in D_{\ell}$. On the other hand, we obtain

$$
\begin{aligned}
\operatorname{Dim}_{\psi}(\xi) & =\sum_{j=1}^{\infty} \sum_{k \in \mathbb{Z}^{d}}\left|\widehat{\psi}\left(D^{j}(\xi+2 \pi k)\right)\right|^{2} \\
& =\sum_{j=1}^{\infty} \sum_{k \in \mathbb{Z}^{d}}\left|\lambda_{j}^{\ell}(\xi)\right|^{2}\left|\widehat{\psi}\left(D^{\ell}(\xi+2 \pi k)\right)\right|^{2} \\
& =\sum_{j=1}^{\infty}\left|\lambda_{j}^{\ell}(\xi)\right|^{2} \sum_{k \in \mathbb{Z}^{d}}\left|\widehat{\psi}\left(D^{\ell}(\xi+2 \pi k)\right)\right|^{2} .
\end{aligned}
$$

Using (4.26), (4.27), and the above equation, we obtain

$$
\sum_{j=1}^{\infty}\left|\widehat{\psi}\left(D^{j} \xi\right)\right|^{2}=\frac{\operatorname{Dim}_{\psi}(\xi)}{\sum_{k \in \mathbb{Z}^{d}}\left|\widehat{\psi}\left(D^{\ell}(\xi+2 \pi k)\right)\right|^{2}}\left|\widehat{\psi}\left(D^{l} \xi\right)\right|^{2}
$$

Combine (4.29) with definition of $\varphi_{0}$, for a.e. $\xi \in D_{\ell}$, we obtain

$$
\left|\widehat{\varphi_{0}}(\xi)\right|^{2}=\sum_{j=1}^{\infty}\left|\widehat{\psi}\left(D^{j} \xi\right)\right|^{2}
$$

This completes the proof of Lemma 4.6 since $z$ and $p_{j}, j \geqslant 1$ cover all of $\mathbb{R}^{d}$.

Lemma 4.7. There exists a $2 \pi-\mathbb{Z}^{d}$ periodic, measurable function $H_{1}: \mathfrak{Z}^{C} \rightarrow \mathbb{C}$ such that

$$
\begin{gathered}
\left|H_{1}(\xi)\right| \leqslant 1, \quad \text { a.e. } \xi \in \mathfrak{Z}^{C}, \\
\widehat{\psi}(D \xi)=e^{i \varepsilon_{0} \cdot \xi} \overline{H_{1}(\xi)} \widehat{\varphi_{0}}(\xi), \quad \text { a.e. } \xi \in \mathfrak{Z}^{C} .
\end{gathered}
$$

Proof. Notice that the $2 \pi-\mathbb{Z}^{d}$ periodicity of $H_{1}$ is consistent with the $2 \pi-\mathbb{Z}^{d}$ periodicity of the measurable set

$$
z^{C}=\bigcup_{\ell \in \mathbb{N}} p_{\ell}
$$


Define $H_{1}$ as the following formula

$$
H_{1}(\xi)= \begin{cases}0, & \xi \in p_{\ell}, l \geqslant 2, \\ e^{i \varepsilon_{0} \cdot \xi} \sqrt{\frac{\sum_{k \in \mathbb{Z}^{d}}|\widehat{\psi}(D(\xi+2 \pi k))|^{2}}{\operatorname{Dim}_{\psi}(\xi)},} & \xi \in p_{1} .\end{cases}
$$

Since all the functions and sets involved in (4.34) are measurable and $2 \pi-\mathbb{Z}^{d}$ periodic, it is obvious that (4.34) defines a $2 \pi-\mathbb{Z}^{d}$ periodic, measurable function $H_{1}: z^{\mathcal{C}} \rightarrow \mathbb{C}$. Furthermore,

$$
\sum_{k \in \mathbb{Z}^{d}}|\widehat{\psi}(D(\xi+2 \pi k))|^{2} \leqslant \operatorname{Dim}_{\psi}(\xi)
$$

implies that (4.31) is valid. For $\xi \in D_{1},(4.32)$ is the direct consequence of (4.21) and (4.34). For $\xi \in D_{\ell}, \ell \geqslant 2, \Psi_{1}(\xi)=0$. In particular, $\widehat{\psi}(D \xi)=0$; thus (4.32) is trivially satisfied. The proof of Lemma 4.7 is completed.

Lemma 4.8. There exists a $2 \pi-\mathbb{Z}^{d}$ periodic, measurable function $H_{0}: \mathfrak{Z}^{\mathcal{C}} \rightarrow \mathbb{C}$ such that

$$
\begin{gathered}
\left|H_{0}(\xi)\right| \leqslant 1, \quad \text { a.e. } \xi \in \mathfrak{Z}^{\mathcal{C}}, \\
\widehat{\varphi_{0}}(D \xi)=H_{0}(\xi) \widehat{\varphi_{0}}(\xi), \quad \text { a.e. } \xi \in \mathcal{Z}^{\mathcal{C}} .
\end{gathered}
$$

Proof. Again, by (4.33), the $2 \pi-\mathbb{Z}^{d}$ periodicity will be clear from the argument, and we need only to prove (4.36) and (4.37) on $\bigcup_{\ell \in \mathbb{N}} p_{\ell}$.

First, we consider $p_{\ell}, \ell \geqslant 2$. For a.e. $\xi \in D_{\ell}$, we obtain

$$
\begin{aligned}
\left\|\Psi_{\ell-1}(D \xi)\right\|_{\ell^{2}\left(\mathbb{Z}^{d}\right)}^{2} & =\sum_{k \in \mathbb{Z}^{d}}\left|\widehat{\psi}\left(D^{\ell-1}(D \xi+2 \pi k)\right)\right|^{2} \\
& =\sum_{k \in \mathbb{Z}^{d}}\left|\widehat{\psi}\left(D^{\ell}\left(\xi+2 \pi D^{-1} k\right)\right)\right|^{2} \\
& \geqslant \sum_{k \in \mathbb{Z}^{d}}\left|\widehat{\psi}\left(D^{\ell}(\xi+2 \pi k)\right)\right|^{2} \\
& =\left\|\Psi_{\ell}(\xi)\right\|_{\ell^{2}\left(\mathbb{Z}^{d}\right)}^{2}>0 .
\end{aligned}
$$

This and the definition (4.20) of $p_{\ell}$ imply that for a.e. $\xi \in p_{\ell}$, there exists $k=k(\xi) \in$ $\{1,2, \ldots, \ell-1\}$ such that $D \xi \in p_{k}$. It is easy to check, using $(4.20)$, that $\xi \rightarrow k(\xi)$ is $2 \pi-\mathbb{Z}^{d}$ periodic and measurable (defined on $D_{\ell}$ ). Since, according to our assumption, $\operatorname{dim} \mathbb{F}_{\psi}(D \xi)=$ 1 for a.e. $\xi \in D_{\ell}$, there exists a $2 \pi$ - $\mathbb{Z}^{d}$ periodic, measurable function $\lambda: D_{\ell} \rightarrow \mathbb{C}$ such that, for a.e. $\xi \in D_{\ell}$,

$$
\Psi_{\ell-1}(D \xi)=\lambda(\xi) \Psi_{k(\xi)}(D \xi)
$$


Notice that $\lambda(\xi) \neq 0$, since otherwise we would have

$$
0=\left\|\Psi_{\ell-1}(D \xi)\right\|_{\ell^{2}\left(\mathbb{Z}^{d}\right)}^{2} \geqslant\left\|\Psi_{\ell}(\xi)\right\|_{\ell^{2}\left(\mathbb{Z}^{d}\right)}^{2} \geqslant 0
$$

which would imply $\Psi_{\ell}(\xi)=0$; this is impossible for $\xi \in D_{\ell}$. Hence, (4.39) implies that, for a.e. $\xi \in D_{\ell}$,

$$
\begin{aligned}
\widehat{\varphi_{0}}(D \xi) & =\sqrt{\frac{\operatorname{Dim}_{\psi}(D \xi)}{\sum_{j \in \mathbb{Z}^{d}}\left|\widehat{\psi}\left(D^{k}(D \xi+2 \pi j)\right)\right|^{2}}} \cdot \widehat{\psi}\left(D^{k} \cdot D \xi\right) \\
& =\sqrt{\frac{\operatorname{Dim}_{\psi}(D \xi)}{\sum_{j \in \mathbb{Z}^{d}}\left|\widehat{\psi}\left(D^{k}(D \xi+2 \pi j)\right)\right|^{2}}} \cdot \frac{1}{\lambda(\xi)} \widehat{\psi}\left(D^{\ell-1} \cdot D \xi\right) .
\end{aligned}
$$

Notice that (4.41) shows that there exists a $2 \pi-\mathbb{Z}^{d}$ periodic, measurable function $\mathscr{A}: p_{\ell} \rightarrow \mathbb{C}$ such that, for a.e. $\xi \in D_{\ell}$,

$$
\widehat{\varphi_{0}}(D \xi)=\mathcal{A}(\xi) \widehat{\psi}\left(D^{\ell} \xi\right)
$$

At the same time it follows directly from (4.21), that there exists a $2 \pi-\mathbb{Z}^{d}$ periodic, measurable function $B: D_{\ell} \rightarrow \mathbb{C}$ such that, for a.e. $\xi \in D_{\ell}, \mathbb{B}(\xi) \neq 0$, and

$$
\widehat{\varphi_{0}}(\xi)=ß(\xi) \widehat{\psi}\left(D^{\ell} \xi\right)
$$

We define $H_{0}(\xi)$ on $p_{\ell}$ by

$$
H_{0}(\xi):=\frac{A(\xi)}{B(\xi)}
$$

By (4.42) and (4.43), it is clear that $H_{0}(\xi)$ is a $2 \pi$ - $\mathbb{Z}^{d}$ periodic, measurable function, and satisfies (4.37) on $p_{\ell}$. Therefore, it remains to define $H_{0}$ on $p_{1}$. Observe that for $\xi \in p_{1}$, either $D \xi \in \mathcal{Z}$, or, otherwise, there is a $2 \pi-\mathbb{Z}^{d}$ periodic, measurable function $\xi \rightarrow \ell(D \xi) \in \mathbb{N}$, such that $D \xi \in P_{\ell(D \xi)}$. If $D \xi \in \mathcal{Z}$, then we define $H_{0}(\xi)$ to be 0 . Otherwise, we define, by (4.21), that there exists a $2 \pi-\mathbb{Z}^{d}$ periodic, measurable function $\tilde{A}$ such that

$$
\widehat{\varphi_{0}}(D \xi)=\tilde{A}(D \xi) \widehat{\psi}\left(D^{\ell(D \xi)} \cdot D \xi\right)=\tilde{A}(D \xi) \widehat{\psi}\left(D^{\ell(D \xi)+1} \xi\right)
$$

Finally, we consider $\xi \in D_{1}$, we know that there exists a $2 \pi$ - $\mathbb{Z}^{d}$ periodic, measurable function $\tilde{\imath}$, such that

$$
\Psi_{\ell(D \xi)+1}(\xi)=\tilde{\lambda}(\xi) \cdot \Psi_{1}(\xi)
$$


and a $2 \pi-\mathbb{Z}^{d}$ periodic, measurable function $\tilde{\mathbb{B}} \neq 0$, such that

$$
\widehat{\varphi_{0}}(\xi)=\widetilde{B}(\xi) \cdot \widehat{\psi}(D \xi) .
$$

Hence, for $\xi \in D_{1}$ and $D \xi \notin \mathcal{Z}$, we define $H_{0}$ by

$$
H_{0}(\xi):=\frac{\tilde{A}(D \xi) \tilde{\lambda}(\xi)}{\widetilde{B}(\xi)}
$$

It is now clear that $H_{0}$ is a $2 \pi-\mathbb{Z}^{d}$ periodic, measurable function on $p_{1}$ and satisfies (4.37) on $p_{1}$. It follows that we have a $2 \pi$ - $\mathbb{Z}^{d}$ periodic, measurable function $H_{0}: Z^{\mathcal{C}} \rightarrow \mathbb{C}$, such that (4.37) is satisfied.

Let us prove (4.36). For $\xi \in \mathbb{Z}^{c}$, we have, by (4.22), that there exists $k \in \mathbb{Z}^{d}$ such that $\widehat{\varphi}_{0}(\xi+2 \pi k) \neq 0$. Since $H_{0}$ is a $2 \pi-\mathbb{Z}^{d}$ periodic we obtain, by (4.37),

$$
\widehat{\varphi_{0}}(D(\xi+2 \pi k))=H_{0}(\xi+2 \pi k) \widehat{\varphi_{0}}(\xi+2 \pi k)=H_{0}(\xi) \widehat{\varphi_{0}}(\xi+2 \pi k) .
$$

Hence

$$
\left|H_{0}(\xi)\right|=\frac{\left|\widehat{\varphi_{0}}(D(\xi+2 \pi k))\right|}{\left|\widehat{\varphi_{0}}(\xi+2 \pi k)\right|}
$$

However, Lemma 4.6 implies that for a.e. $\mu \in \mathbb{R}^{d},\left|\widehat{\varphi_{0}}(D \mu)\right|^{2} \leqslant\left|\widehat{\varphi}_{0}(\mu)\right|^{2}$. Thus, $\left|H_{0}(\xi)\right| \leqslant 1$, and this completes the proof of Lemma 4.8.

Now, let us extend the definition of $H_{0}$ and $H_{1}$ to $\mathcal{z}$, as well. Since $H_{0}$ is already defined on $z^{C}$, the following definition of $H_{1}$ on $z$ makes sense:

$$
H_{1}(\xi)= \begin{cases}\frac{1}{\sqrt{2}}, & \xi \in \mathfrak{Z}, \xi+2 \pi D^{-1} \varepsilon_{0} \in \mathfrak{Z}, \\ H_{0}\left(\xi+2 \pi D^{-1} \varepsilon_{0}\right), & \xi \in \mathcal{Z}, \xi+2 \pi D^{-1} \varepsilon_{0} \notin \mathcal{Z} .\end{cases}
$$

Using Lemmas 4.7 and 4.8 and (4.51), we obtain that $H_{1}: \mathbb{R}^{d} \rightarrow \mathbb{C}$ is a $2 \pi$ - $\mathbb{Z}^{d}$ periodic, measurable function, such that

$$
\left|H_{1}(\xi)\right| \leqslant 1, \quad \text { a.e. } \xi \in \mathbb{R}^{d}
$$

Moreover, we conclude that (4.32) is now satisfied for a.e. $\xi \in \mathbb{R}^{d}$. By Lemma 4.7, we need to check (4.32) only on $z$. But, for $\xi \in \mathcal{z}$, we have that $\widehat{\varphi_{0}}(\xi)=0$, by $(4.21)$, and $\widehat{\psi}(D \xi)=0$, since $\operatorname{Dim}_{\psi}(\xi)=0$. Hence, on $\mathcal{Z}(4.32)$ is satisfied irrespective of the value on $H_{1}(\xi)$. We have established, that

$$
\widehat{\psi}(D \xi)=e^{i \varepsilon_{0} \cdot \xi} \overline{H_{1}(\xi)} \widehat{\varphi_{0}}(\xi), \quad \text { a.e. } \xi \in \mathcal{Z}^{C} .
$$


Let us turn our attention to $H_{0}$. Now that $H_{1}$ is defined on all of $\mathbb{R}^{d}$, we define $H_{0}$ on zby

$$
H_{0}(\xi):=H_{1}\left(\xi+2 \pi D^{-1} \varepsilon_{0}\right), \quad \text { a.e. } \xi \in \mathbb{R}^{d} .
$$

Obviously, $H_{0}: \mathbb{R} \rightarrow \mathbb{C}$ is a $2 \pi-\mathbb{Z}^{d}$ periodic, measurable function, such that

$$
\left|H_{0}(\xi)\right| \leqslant 1, \quad \text { a.e. } \xi \in \mathbb{R}^{d}
$$

Again, we obtain that such $H_{0}$ satisfies (4.37) on $\mathbb{R}^{d}$. And, again, because of Lemma 4.8, it is enough to check (4.37) on $z$. Since $\widehat{\varphi}_{0}(\xi)=0$, for $\xi \in z$, it is enough to show that $\widehat{\varphi}_{0}(D \xi)=0$, for $\xi \in$ z. But this is an immediate consequence of Lemma 4.6. We conclude that, for a.e. $\xi \in \mathbb{R}$,

$$
\widehat{\varphi}_{0}(D \xi)=H_{0}(\xi) \widehat{\varphi}_{0}(\xi)
$$

The following lemma connects $H_{0}$ and $H_{1}$.

Lemma 4.9. For a.e. $\xi \in \mathbb{R}^{d}$

$$
\left|H_{0}(\xi)\right|^{2}+\left|H_{1}(\xi)\right|^{2}=1
$$

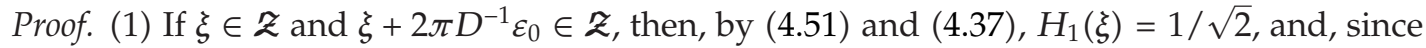
$\xi+4 \pi D^{-1} \varepsilon_{0} \in \mathcal{Z}$, so

$$
H_{0}(\xi)=H_{1}\left(\xi+2 \pi D^{-1} \varepsilon_{0}\right)=\frac{1}{\sqrt{2}} .
$$

Obviously, (4.57) is satisfied.

(2) If $\xi \in \mathcal{Z}$, and $\xi+2 \pi D^{-1} \varepsilon_{0} \notin \mathcal{Z}$, then by (4.51) and (4.54),

$$
\left|H_{0}(\xi)\right|^{2}+\left|H_{1}(\xi)\right|^{2}=\left|H_{0}\left(\xi+2 \pi D^{-1} \varepsilon_{0}\right)\right|^{2}+\left|H_{1}\left(\xi+2 \pi D^{-1} \varepsilon_{0}\right)\right|^{2}
$$

Hence, (4.57) is going to be satisfied if we can prove it on $\mathfrak{Z}^{\mathcal{C}}$. We present this argument. Lemma 4.6 implies that, for a.e. $\xi \in \mathbb{R}^{d}$

$$
\left|\widehat{\varphi}_{0}(\xi)\right|^{2}=|\widehat{\psi}(D \xi)|^{2}+\left|\widehat{\varphi}_{0}(D \xi)\right|^{2}
$$

From (4.53) and (4.56) we obtain that, for a.e. $\xi \in \mathbb{R}^{d}$

$$
\left|\widehat{\varphi}_{0}(\xi)\right|^{2}=\left[\left|H_{0}(\xi)\right|^{2}+\left|H_{1}(\xi)\right|^{2}\right] \cdot\left|\widehat{\varphi}_{0}(\xi)\right|^{2} .
$$


Using the fact that $H_{0}(\xi)$ and $H_{1}(\xi)$ are $2 \pi-\mathbb{Z}^{d}$ periodic, we can periodize (4.61). This periodization and (4.22) provides us with

$$
\operatorname{Dim}_{\psi}(\xi)=\left[\left|H_{0}(\xi)\right|^{2}+\left|H_{1}(\xi)\right|^{2}\right] \cdot \operatorname{Dim}_{\psi}(\xi)
$$

Recall that on $z^{\mathcal{C}}, \operatorname{Dim}_{\Psi}(\xi) \neq 0$; thus (4.62) provides us with (4.57) on $z^{\mathcal{C}}$.

The following lemma establishes that $H_{0}(\xi)$ and $H_{1}(\xi)$ are generalized filters, and provides the crucial step to find $\rho$ such that (4.18) is satisfied. It is interesting to observe that the proof of this lemma relies on the fact that $\rho_{q}(\xi)=0, q \in \mathbb{S}$.

Lemma 4.10. For a.e. $\xi \in \mathbb{R}^{d}$

$$
\begin{gathered}
H_{0}(\xi) \overline{H_{0}\left(\xi+2 \pi D^{-1} \varepsilon_{0}\right)}=H_{1}\left(\xi+2 \pi D^{-1} \varepsilon_{0}\right) \overline{H_{1}(\xi)} \\
\left|H_{0}(\xi)\right|=\left|H_{1}\left(\xi+2 \pi D^{-1} \varepsilon_{0}\right)\right| \\
\left|H_{0}(\xi)\right|^{2}+\left|H_{0}\left(\xi+2 \pi D^{-1} \varepsilon_{0}\right)\right|^{2}=\left|H_{1}(\xi)\right|^{2}+\left|H_{1}\left(\xi+2 \pi D^{-1} \varepsilon_{0}\right)\right|^{2}=1 .
\end{gathered}
$$

Proof. We only need to prove (4.63). Indeed, (4.63) and Lemma 4.9 imply that

$$
\begin{aligned}
\left|H_{0}(\xi)\right|^{2} & =\left|H_{0}(\xi)\right|^{2} \cdot\left|H_{0}\left(\xi+2 \pi D^{-1} \varepsilon_{0}\right)\right|^{2}+\left|H_{0}(\xi)\right|^{2} \cdot\left|H_{1}\left(\xi+2 \pi D^{-1} \varepsilon_{0}\right)\right|^{2} \\
& =\left|H_{1}(\xi)\right|^{2} \cdot\left|H_{1}\left(\xi+2 \pi D^{-1} \varepsilon_{0}\right)\right|^{2}+\left|H_{0}(\xi)\right|^{2} \cdot\left|H_{1}\left(\xi+2 \pi D^{-1} \varepsilon_{0}\right)\right|^{2} \\
& =\left|H_{1}\left(\xi+2 \pi D^{-1} \varepsilon_{0}\right)\right|^{2} .
\end{aligned}
$$

This clearly implies (4.64). Lemma 4.9 and (4.64) imply (4.65) straightforwardly.

Let us prove (4.63). First, we consider $\xi \in \mathfrak{Z} \bigcup\left(\mathfrak{Z}+2 \pi D^{-1} \varepsilon_{0}\right)$. We have three cases.

(1) If $\xi \in \mathcal{z}$ and $\xi+2 \pi D^{-1} \varepsilon_{0} \notin z$. By (4.51) and (4.54), we have

$$
H_{0}(\xi)=H_{1}\left(\xi+2 \pi D^{-1} \varepsilon_{0}\right), \quad H_{1}(\xi)=H_{0}\left(\xi+2 \pi D^{-1} \varepsilon_{0}\right)
$$

which clearly establishes (4.63).

(2) If $\xi \in \mathcal{Z}$ and $\xi+2 \pi D^{-1} \varepsilon_{0} \in \mathcal{Z}$. By (4.51) and (4.54), we have

$$
H_{0}(\xi)=H_{1}\left(\xi+2 \pi D^{-1} \varepsilon_{0}\right), \quad H_{1}(\xi)=H_{0}\left(\xi+2 \pi D^{-1} \varepsilon_{0}\right)=\frac{1}{\sqrt{2}} .
$$

Again, (4.63) follows. 

have,

(3) If $\xi \notin \mathcal{z}$ and $\xi+2 \pi D^{-1} \varepsilon_{0} \in \mathcal{z}$, then $\xi+4 \pi D^{-1} \varepsilon_{0} \notin \mathcal{Z}$, thus, by (4.51) and (4.54), we

$$
H_{1}\left(\xi+2 \pi D^{-1} \varepsilon_{0}\right)=H_{0}\left(\xi+4 \pi D^{-1} \varepsilon_{0}\right)=H_{0}(\xi), \quad H_{0}\left(\xi+2 \pi D^{-1} \varepsilon_{0}\right)=H_{1}(\xi) .
$$

Obviously, (4.63) follows.

Finally, let us consider $\xi \in\left[z \cup\left(z+2 \pi D^{-1} \varepsilon_{0}\right)\right]^{\complement}$. Using (4.53), (4.56), and (4.57), we obtain, for $j \geqslant 1, q \in \mathbb{S}$, and a.e. $\zeta \in \mathbb{R}^{d}$,

$$
\widehat{\psi}\left(D^{j} \zeta\right) \overline{\widehat{\psi}\left(D^{j}(\zeta+2 \pi q)\right)}=\widehat{\varphi_{0}}\left(D^{j-1} \zeta\right) \overline{\widehat{\varphi_{0}}\left(D^{j-1}(\zeta+2 \pi q)\right)}-\widehat{\varphi_{0}}\left(D^{j} \zeta\right) \overline{\widehat{\varphi_{0}}\left(D^{j}(\zeta+2 \pi q)\right)} .
$$

By Proposition 2.2, for $q \in \mathbb{S}$, and a.e. $\zeta \in \mathbb{R}^{d}$, we obtain

$$
\begin{aligned}
0= & \rho_{q}(\zeta)=\sum_{j=0}^{+\infty} \widehat{\psi}\left(D^{j} \zeta\right) \overline{\widehat{\psi}\left(D^{j}(\zeta+2 \pi q)\right)} \\
= & \widehat{\psi}(\zeta) \overline{\widehat{\psi}(\zeta+2 \pi q)}+\widehat{\varphi_{0}}(\zeta) \overline{\widehat{\varphi_{0}}(\zeta+2 \pi q)} \\
= & {\left[H_{0}\left(D^{-1} \zeta\right) \overline{H_{0}\left(D^{-1} \zeta+2 \pi D^{-1} \varepsilon_{0}\right)}-\overline{H_{1}\left(D^{-1} \zeta\right)} H_{1}\left(D^{-1} \zeta+2 \pi D^{-1} \varepsilon_{0}\right)\right] } \\
& \cdot \widehat{\varphi_{0}}\left(D^{-1} \zeta\right) \overline{\widehat{\varphi_{0}}\left(D^{-1} \zeta+2 \pi D^{-1} q\right)} .
\end{aligned}
$$

Now suppose $\xi \notin \mathcal{Z}$ and $\xi+2 \pi D^{-1} \varepsilon_{0} \notin \mathcal{Z}$. By (4.22) there exist $k, \ell \in \mathbb{Z}^{d}$ such that

$$
\widehat{\varphi_{0}}(\xi+2 \pi k) \neq 0, \quad \widehat{\varphi_{0}}\left(\xi+2 \pi \ell+2 \pi D^{-1} \varepsilon_{0}\right) \neq 0
$$

Let

$$
D^{-1} \zeta=\xi+2 \pi k, \quad q=D(\ell-k)+\varepsilon_{0}
$$

and we conclude

$$
\begin{aligned}
0 & =H_{0}(\xi+2 \pi k) \overline{H_{0}\left(\xi+2 \pi D^{-1} \varepsilon_{0}\right)}-\overline{H_{1}(\xi+2 \pi k)} H_{1}\left(\xi+2 \pi D^{-1} \varepsilon_{0}\right) \\
& =H_{0}(\xi) \overline{H_{0}\left(\xi+2 \pi D^{-1} \varepsilon_{0}\right)}-\overline{H_{1}(\xi)} H_{1}\left(\xi+2 \pi D^{-1} \varepsilon_{0}\right) .
\end{aligned}
$$

This proves the case $\xi \in\left[\mathcal{Z}\left(\mathfrak{Z}+2 \pi D^{-1} \varepsilon_{0}\right)\right]^{\complement}$ and completes the proof of Lemma 4.10.

We have established so far that $H_{0}$ and $H_{1}$ are generalized filters, and that $\varphi_{0}$ is a pseudoscaling function and that $H_{0}$ is its corresponding filter, and that $\widehat{\psi}$ satisfies (4.53). We will now prove that (4.53) provides us with (4.21), which is going to complete the proof of the Theorem 4.5. 
Let

$$
\Omega=\left\{\xi \in \mathbb{R}^{d}: H_{0}\left(\xi+2 \pi D^{-1} \varepsilon_{0}\right)=0\right\} .
$$

It is clear that both $\Omega$ and $\Omega^{C}$ are measurable and $2 \pi$ - $\mathbb{Z}^{d}$ periodic. Furthermore, by (4.65), we have

$$
\xi \in \Omega \longrightarrow \xi+2 \pi D^{-1} \varepsilon_{0} \in \Omega^{C}, \quad \text { a.e. }
$$

For $\xi \in \Omega^{C}$, define a function $\mathcal{S}$ as follows:

$$
\mathcal{S}(\xi):=\frac{H_{1}(\xi)}{H_{0}\left(\xi+2 \pi D^{-1} \varepsilon_{0}\right)}
$$

By (4.64), it follows that $S: \Omega^{C} \rightarrow \mathbb{C}$ is a $2 \pi$ - $\mathbb{Z}^{d}$ periodic, unimodular function. For $\xi \in \Omega$, we use (4.76) to conclude that $\xi+2 \pi D^{-1} \varepsilon_{0} \in \Omega^{\mathcal{C}}$, and we define $\mathcal{S}$ by

$$
\mathcal{S}(\xi):=\mathcal{S}\left(\xi+2 \pi D^{-1} \varepsilon_{0}\right)
$$

Hence, $\mathcal{S}(\xi)$ is an unimodular, $2 \pi-\mathbb{Z}^{d}$ periodic function on $\mathbb{R}^{d}$, which satisfies

$$
H_{1}(\xi)=\mathcal{S}(\xi) H_{0}\left(\xi+2 \pi D^{-1} \varepsilon_{0}\right), \quad \text { a.e. }
$$

Lemma 4.11. $\mathcal{S}(\xi)$ is a $\pi-\mathbb{Z}^{d}$ periodic function.

Proof. Firstly, if $\xi \in \mathbb{R}^{d}$ such that $H_{0}(\xi) \overline{H_{0}\left(\xi+2 \pi D^{-1} \varepsilon_{0}\right)}=0$. By (4.76), we have two possibilities: $\xi \in \Omega$ and $\xi+2 \pi D^{-1} \varepsilon_{0} \notin \Omega$, or $\xi \notin \Omega$ and $\xi+2 \pi D^{-1} \varepsilon_{0} \in \Omega$. In both cases (4.78) and $2 \pi-\mathbb{Z}^{d}$ periodic $\mathcal{S}(\xi)$ provide us with the conclusion that $\mathcal{S}(\xi)=\mathcal{S}\left(\xi+2 \pi D^{-1} \varepsilon_{0}\right)$.

Secondly, if $\xi \in \mathbb{R}^{d}$ such that $H_{0}(\xi) \overline{H_{0}\left(\xi+2 \pi D^{-1} \varepsilon_{0}\right)} \neq 0$. In this case, we apply (4.63), (4.79), and the unimodularity of $\mathcal{S}(\xi)$ to obtain

$$
\begin{aligned}
H_{0}(\xi) \overline{H_{0}\left(\xi+2 \pi D^{-1} \varepsilon_{0}\right)} & =\overline{H_{1}(\xi)} H_{1}\left(\xi+2 \pi D^{-1} \varepsilon_{0}\right) \\
& =\mathcal{S}\left(\xi+2 \pi D^{-1} \varepsilon_{0}\right) H_{0}(\xi) \overline{\mathcal{S}(\xi) H_{0}\left(\xi+2 \pi D^{-1} \varepsilon_{0}\right)} \\
& =\left\{\mathcal{S}\left(\xi+2 \pi D^{-1} \varepsilon_{0}\right) \overline{\mathcal{S}(\xi)}\right\} H_{0}(\xi) \overline{H_{0}\left(\xi+2 \pi D^{-1} \varepsilon_{0}\right)}
\end{aligned}
$$

Therefore,

$$
\mathcal{S}(\xi)=\mathcal{S}\left(\xi+2 \pi D^{-1} \varepsilon_{0}\right)
$$

The proof of Lemma 4.11 is completed. 
For $\xi \in \mathbb{R}^{d}$, let

$$
\rho(\xi)=\overline{\mathcal{S}\left(D^{-1} \xi\right)}
$$

Since $\mathcal{S}(\xi)$ is a $\pi-\mathbb{Z}^{d}$ periodic, unimodular function, so $\rho(\xi)$ is a $2 \pi-\mathbb{Z}^{d}$ periodic, unimodular function. Hence, $H_{0}, \varphi_{0}$ and $\rho(\xi)$ satisfy all the necessary requirements. It remains to check that they satisfy (4.18), as well. Indeed, by (4.53), (4.79), and (4.82), we obtain

$$
\widehat{\psi}(D \xi)=e^{i \varepsilon_{0} \cdot \xi} \overline{H_{1}(\xi)} \widehat{\varphi_{0}}(\xi)=e^{i \varepsilon_{0} \cdot \xi} \rho(D \xi) \overline{H_{0}\left(\xi+2 \pi D^{-1} \varepsilon_{0}\right)} \widehat{\varphi_{0}}(\xi) .
$$

The proof of Theorem 4.5 is completed.

Theorem 4.12. Suppose that $\psi$ is a M-TFW. Then, $\psi$ is a semi-orthogonal MRA M-TFW if and only if

$$
\operatorname{Dim}_{\psi}(\xi) \in\{0,1\}, \quad \text { for a.e. } \xi \in \mathbb{R}^{d}
$$

Proof. Suppose that $\psi$ is a semi-orthogonal MRA M-TFW, using Theorems 4.1 and 4.5 , then (4.84) is valid.

Suppose that $\psi$ is a M-TFW and (4.84) is valid. By Theorem 4.1, we conclude that $\psi$ is semi-orthogonal, and, thus, by (4.13), $\operatorname{Dim}_{\psi}(\xi) \in\{0,1\}$, for a.e. $\xi \in \mathbb{R}^{d}$. By Theorem 4.5 , we conclude that $\psi$ is an MRA M-TFW.

\section{Conclusions}

In this paper, we study all generalized low-pass filters and M-TFW. Firstly, we study the pseudoscaling function, generalized low-pass filters, and MRA M-TFW and give some important characterizations about them. Then, we characterize all M-TFW by showing that they correspond precisely to those of which the dimension function is nonnegative integer valued. Finally, we also show that an M-TFW arises from our MRA construction if and only if the dimension of a particular linear space is either zero or one.

\section{Acknowledgments}

The authors would like to express their gratitude to the referee for his (or her) valuable comments and suggestions. This work was supported by the National Natural Science Foundation of China (Grant no. 10961001), the Key Project of Chinese Ministry of Eduction (Grant no. 209152), and the Science Foundation of Ningxia higher School (no. 2010JY003).

\section{References}

[1] I. Daubechies, Ten Lectures on Wavelets, vol. 61 of CBMS-NSF Regional Conference Series in Applied Mathematics, SIAM, Philadelphia, Pa, USA, 1992.

[2] L. Debnath, Wavelet Transforms and Their Applications, Birkhäuser, Boston, Mass, USA, 2002.

[3] E. Hernández and G. Weiss, A First Course on Wavelets, Studies in Advanced Mathematics, CRC Press, Boca Raton, Fla, USA, 1996. 
[4] F. Keinert, Wavelets and Multiwavelets, Studies in Advanced Mathematics, Chapman \& Hall/CRC, Boca Raton, Fla, USA, 2004.

[5] O. Christensen, An Introduction to Frames and Riesz Bases, Applied and Numerical Harmonic Analysis, Birkhäuser, Boston, Mass, USA, 2003.

[6] J. J. Benedetto and S. Li, "The theory of multiresolution analysis frames and applications to filter banks," Applied and Computational Harmonic Analysis, vol. 5, no. 4, pp. 389-427, 1998.

[7] J. J. Benedetto and O. M. Treiber, "Wavelet frames: multiresolution analysis and extension principles," in Wavelet Transforms and Time-Frequency Signal Analysis, L. Debnath, Ed., Applied and Numerical Haimonic Analysis, pp. 3-36, Birkhäuser, Boston, Mass, USA, 2001.

[8] M. Bownik, "A characterization of affine dual frames in $L^{2}\left(R^{n}\right)$, " Applied and Computational Harmonic Analysis, vol. 8, no. 2, pp. 203-221, 2000.

[9] D. Bakić, I. Krishtal, and E. N. Wilson, "Parseval frame wavelets with $E_{n}^{(2)}$-dilations," Applied and Computational Harmonic Analysis, vol. 19, no. 3, pp. 386-431, 2005.

[10] D. Bakić, "Semi-orthogonal Parseval frame wavelets and generalized multiresolution analyses," Applied and Computational Harmonic Analysis, vol. 21, no. 3, pp. 281-304, 2006.

[11] M. Bownik, Z. Rzeszotnik, and D.n Speegle, "A characterization of dimension functions of wavelets," Applied and Computational Harmonic Analysis, vol. 10, no. 1, pp. 71-92, 2001.

[12] C. K. Chui, W. He, J. Stöckler, and Q. Sun, "Compactly supported tight affine frames with integer dilations and maximum vanishing moments," Advances in Computational Mathematics, vol. 18, no. 2-4, pp. 159-187, 2003.

[13] I. Daubechies, B. Han, A. Ron, and Z. Shen, "Framelets: MRA-based constructions of wavelet frames," Applied and Computational Harmonic Analysis, vol. 14, no. 1, pp. 1-46, 2003.

[14] M. Ehler, "On multivariate compactly supported bi-frames," The Journal of Fourier Analysis and Applications, vol. 13, no. 5, pp. 511-532, 2007.

[15] B. Han and Q. Mo, "Multiwavelet frames from refinable function vectors," Advances in Computational Mathematics, vol. 18, no. 2-4, pp. 211-245, 2003.

[16] Y. Huang and Z. Cheng, "Minimum-energy frames associated with refinable function of arbitrary integer dilation factor," Chaos, Solitons and Fractals, vol. 32, no. 2, pp. 503-515, 2007.

[17] H. O. Kim and J. K. Lim, "On frame wavelets associated with frame multiresolution analysis," Applied and Computational Harmonic Analysis, vol. 10, no. 1, pp. 61-70, 2001.

[18] M.-J. Lai and J. Stöckler, "Construction of multivariate compactly supported tight wavelet frames," Applied and Computational Harmonic Analysis, vol. 21, no. 3, pp. 324-348, 2006.

[19] S. Li, "A theory of generalized multiresolution structure and pseudoframes of translates," The Journal of Fourier Analysis and Applications, vol. 7, no. 1, pp. 23-40, 2001.

[20] M. Paluszyński, H. Šikić, G. Weiss, and S. Xiao, "Generalized low pass filters and MRA frame wavelets," The Journal of Geometric Analysis, vol. 11, no. 2, pp. 311-342, 2001.

[21] M. Paluszyński, H. Šikić, G. Weiss, and S. Xiao, "Tight frame wavelets, their dimension functions, MRA tight frame wavelets and connectivity properties," Advances in Computational Mathematics, vol. 18, no. 2-4, pp. 297-327, 2003.

[22] A. Ron and Z. Shen, "Affine systems in $L^{2}\left(R^{d}\right)$ : the analysis of the analysis operator," Journal of Functional Analysis, vol. 148, no. 2, pp. 408-447, 1997.

[23] A. Ron and Z. Shen, "Affine systems in $L^{2}\left(R^{d}\right)$. II. Dual systems," The Journal of Fourier Analysis and Applications, vol. 3, no. 5, pp. 617-637, 1997.

[24] H. Sikić, D. Speegle, and G. Weiss, "Structure of the set of dyadic PFW's," in Frames and operator theory in analysis and signal processing, vol. 451 of Contemporary Mathematics, pp. 263-291, American Mathematical Society, Providence, RI, USA, 2008.

[25] G. Wu, X. Yang, and Z. Liu, "MRA Parseval frame wavelets and their multipliers in $L^{2}\left(R^{n}\right)$," Mathematical Problems in Engineering, Article ID 492585, 17 pages, 2009. 


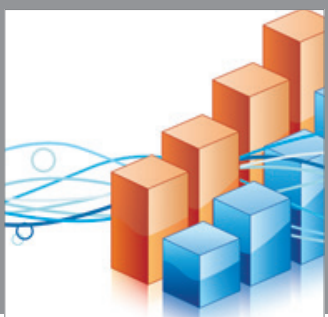

Advances in

Operations Research

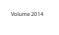

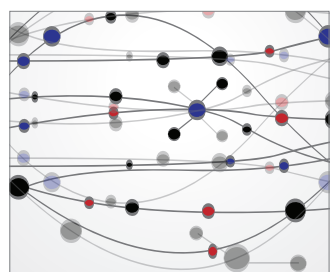

\section{The Scientific} World Journal
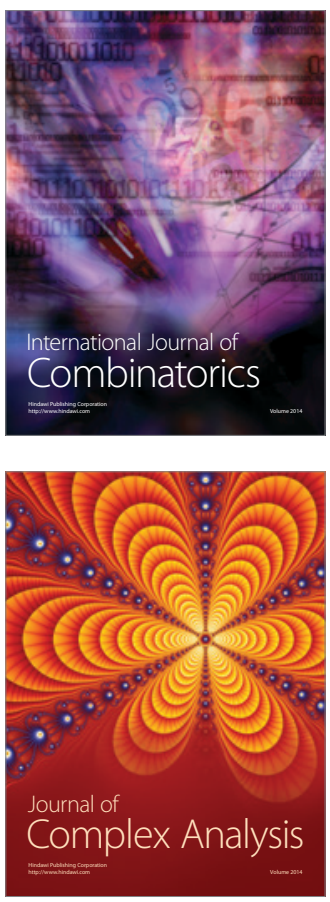

International Journal of

Mathematics and

Mathematical

Sciences
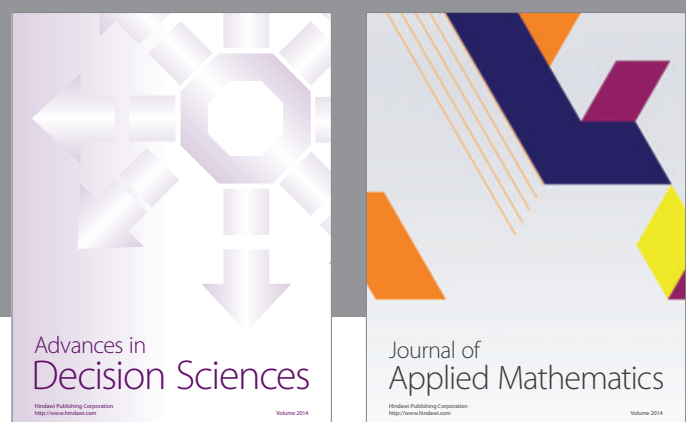

Journal of

Applied Mathematics
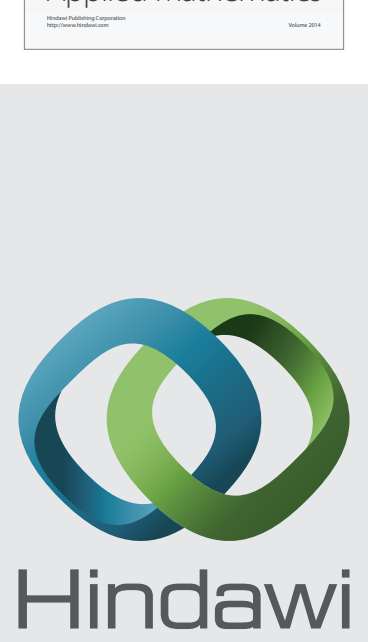

Submit your manuscripts at http://www.hindawi.com
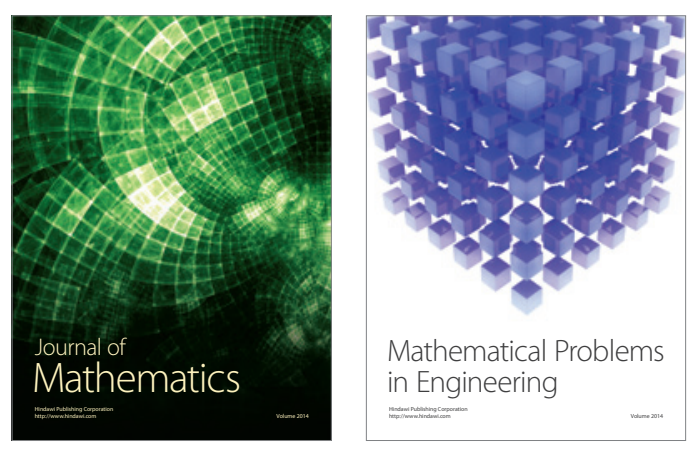

Mathematical Problems in Engineering
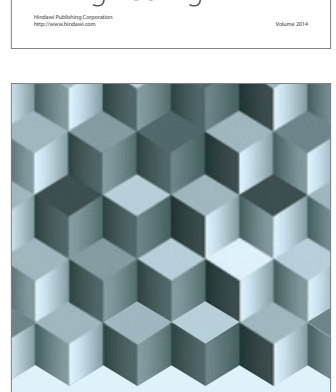

Journal of

Function Spaces
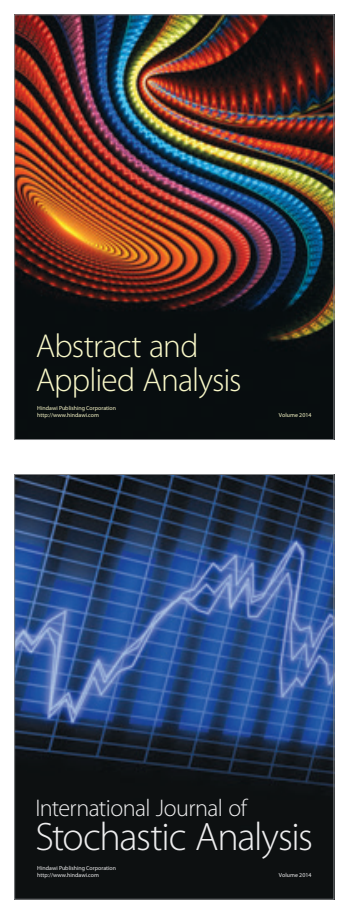

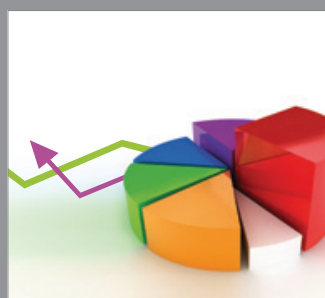

ournal of

Probability and Statistics

Promensencen
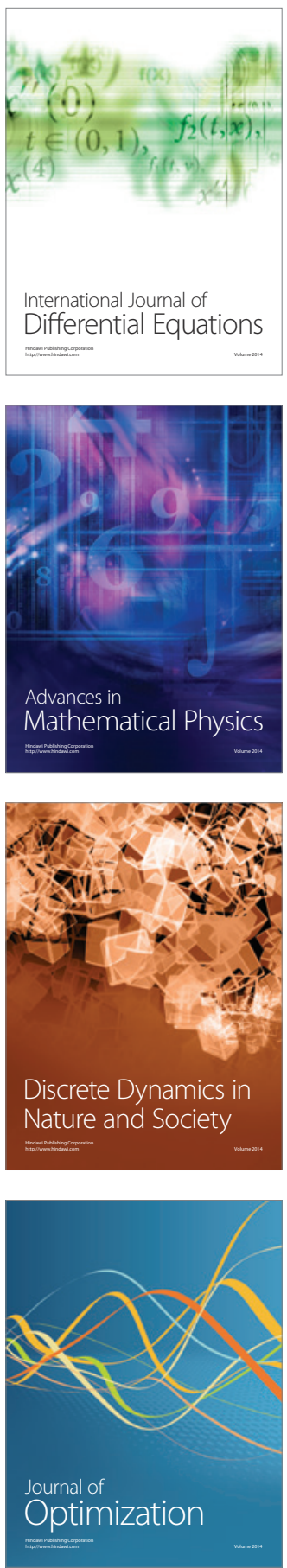